\title{
Granulocyte macrophage colony-stimulating factor induces CCL17 production via IRF4 to mediate inflammation
}

\author{
Adrian Achuthan, ${ }^{1}$ Andrew D. Cook, ${ }^{1}$ Ming-Chin Lee, ${ }^{1}$ Reem Saleh, ${ }^{1}$ Hsu-Wei Khiew, ${ }^{1}$ Melody W.N. Chang, ${ }^{1}$ Cynthia Louis, ${ }^{1}$ \\ Andrew J. Fleetwood, ${ }^{1}$ Derek C. Lacey, ${ }^{1,2,3}$ Anne D. Christensen, ${ }^{1}$ Ashlee T. Frye, ${ }^{1}$ Pui Yeng Lam, ${ }^{1}$ Hitoshi Kusano, ${ }^{1}$ Koji Nomura, ${ }^{1,4}$ \\ Nancy Steiner, ${ }^{5}$ Irmgard Förster, ${ }^{5}$ Stephen L. Nutt, ${ }^{2,3}$ Moshe Olshansky, ${ }^{6}$ Stephen J. Turner, ${ }^{6}$ and John A. Hamilton ${ }^{1}$ \\ 'Department of Medicine, Royal Melbourne Hospital, University of Melbourne, Parkville, Victoria, Australia. ${ }^{2}$ The Walter and Eliza Hall Institute of Medical Research, Parkville, Victoria, Australia. \\ ${ }^{3}$ Department of Medical Biology, University of Melbourne, Parkville, Victoria, Australia. ${ }^{4}$ Department of Orthopedic Surgery, Osaka University, Osaka, Japan. ${ }^{5}$ mmmunology and Environment, \\ Life and Medical Sciences Institute, University of Bonn, Bonn, Germany. 'Department of Microbiology and Immunology, The Peter Doherty Institute for Infection and Immunity, \\ University of Melbourne, Parkville, Victoria, Australia.
}

Data from preclinical and clinical studies have demonstrated that granulocyte macrophage colony-stimulating factor (CMCSF) can function as a key proinflammatory cytokine. However, therapies that directly target GM-CSF function could lead to undesirable side effects, creating a need to delineate downstream pathways and mediators. In this work, we provide evidence that GM-CSF drives CCL17 production by acting through an IFN regulatory factor 4-dependent (IRF4-dependent) pathway in human monocytes, murine macrophages, and mice in vivo. In murine models of arthritis and pain, IRF4 regulated the formation of CCL17, which mediated the proinflammatory and algesic actions of GM-CSF. Mechanistically, GM-CSF upregulated IRF4 expression by enhancing JMJD3 demethylase activity. We also determined that CCL17 has chemokineindependent functions in inflammatory arthritis and pain. These findings indicate that CM-CSF can mediate inflammation and pain by regulating IRF4-induced CCL17 production, providing insights into a pathway with potential therapeutic avenues for the treatment of inflammatory diseases and their associated pain.

\section{Introduction}

In addition to its role as a proinflammatory cytokine, granulocyte macrophage colony-stimulating factor (GM-CSF) has also been implicated in bone cancer pain and in inflammatory and arthritic pain (1-4). Cells of the monocyte/macrophage lineage are likely cell types involved in these functions, with other proinflammatory cytokines, such as TNF, IL-1 $\beta$, IL-23, and IL-6, being implicated in possible feedback loops $(5,6)$; GM-CSF can have a role in the biology of monocyte-derived DCs (moDCs) in autoimmune/inflammatory reactions $(7,8)$.

The chemokine CCL17 (also called thymus and activation-regulated chemokine [TARC]) was originally implicated in the preferential attraction of Th2 lymphocytes and thus considered an M2 cytokine (9); however, it can also attract effector/ memory Th1 lymphocytes and Tregs $(9,10)$. It can be produced by certain macrophage/DC populations $(9,11-13)$; with wide receptor (CCR4) distribution (14), including in neurons (15), CCL17 likely has other properties $(9,12,13,16)$ and is elevated in many inflammatory conditions $(13,16-20)$.

IFN regulatory factor 4 (IRF4) is a hemopoietic-specific transcription factor critical for myeloid and lymphoid lineage devel-

Authorship note: A. Achuthan and A.D. Cook contributed equally to this work. Conflict of interest: The authors have declared that no conflict of interest exists. Submitted: March 30, 2016; Accepted: June 23, 2016.

Reference information: J Clin Invest. 2016;126(9):3453-3466. doi:10.1172/JCI87828. opment and function (21). While an important role for IRF4 in DC development has been emphasized (22-25), in macrophages (26-28) and DCs (29), it has even been considered to provide an antiinflammatory signal. While IRF5 has been proposed to be key for GM-CSF-dependent M1 macrophage polarization (30), we have observed a more dramatic upregulation of IRF4 in GM-CSFtreated monocytes/macrophages than in the corresponding macrophage-CSF-treated (M-CSF-treated) populations (31).

Since the targeting of GM-CSF and its receptor for treatment of inflammatory/autoimmune diseases could have unwanted side effects, such as infections and pulmonary alveolar proteinosis (4, 5), the elucidation of critical downstream pathways/mediators could be advantageous. We provide evidence for a GM-CSFdependent pathway in monocytes/macrophages leading to IRF4-dependent CCL17 formation and for its relevance to inflammation and its associated pain.

\section{Results}

GM-CSF dramatically upregulates CCL17 expression in human monocytes and murine macrophages. Human monocytes were cultured for 16 hours in GM-CSF and gene expression examined by microarray analysis. CCL17 is the most highly upregulated gene among the cytokines/chemokines whose gene expression was significantly altered (Figure 1A and Supplemental Table 1; supplemental material available online with this article; doi:10.1172/JCI87828DS1); the key proinflammatory cytokines, IL1B and TNF, were also upregu- 
A

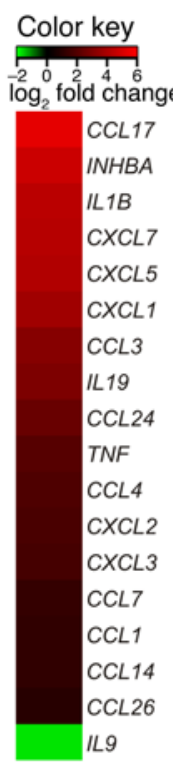

B
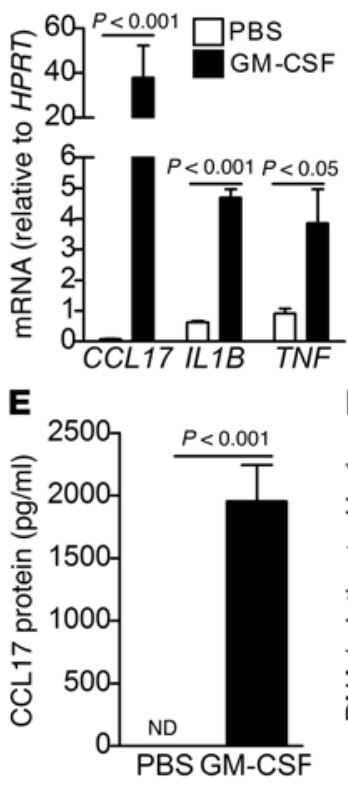

C

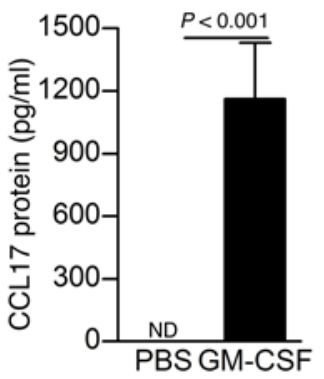

$\mathbf{F}$

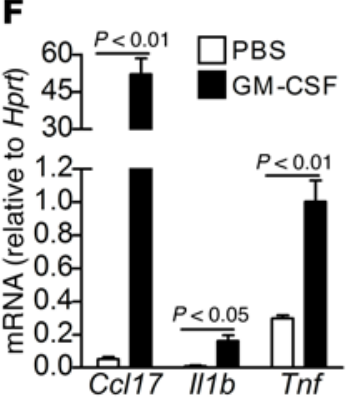

D

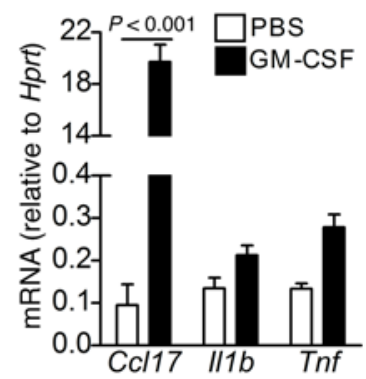

By contrast, Illb and Tnf mRNA were similarly expressed in the respective footpads. Intraplantar (i.pl.) GM-CSF injection further upregulated Ccl17 mRNA expression (Supplemental Figure 2B); $I l 1 b$ and Tnf mRNA expression were also increased, but to a relatively lesser extent.

Ccl17 mRNA was barely detectable in naive joints of WT mice (Supplemental Figure 2C). An intraarticular (i.a.) injection of methylated BSA (mBSA), which leads to a mild synovitis (35), led to Ccl17 mRNA expression that was GM-CSF dependent (Supplemental Figure 2C); Ccl17 mRNA expression was also not detected in the naive or salineinjected joints of $\mathrm{GMCSF}^{-1-}$ mice. WT mice, which also received s.c. GM-CSF (3), showed a further significant increase in $\mathrm{Ccl17}$ mRNA expression, as well as in Illb and Tnf mRNA expression, in the mBSA-injected joints compared with the saline-injected joints (Supplemental Figure 2D).

lated (Figure 1A and Supplemental Table 1). The increased expression of these genes was validated by quantitative PCR (qPCR) (Figure 1B). Unlike IL-1 $\beta$ and TNF, which were not detected in the GM-CSF-treated cultures (data not shown) $(32,33)$, high levels of CCL17 were found in the supernatant (Figure 1C).

For M-CSF-starved (CSF-1-starved) murine bone marrowderived macrophages (BMM), GM-CSF dramatically upregulated Ccl17 mRNA (Figure 1D) and protein (Figure 1E); Il1b and Tnf were not significantly upregulated by GM-CSF, either at the mRNA level (Figure 1D) or as secreted protein (data not shown). Furthermore, by $\mathrm{qPCR}, \mathrm{GM}-\mathrm{CSF}$-differentiated bone marrow cells, here and elsewhere $(33,34)$ termed GM-BMM (see below), had significantly increased levels of Ccl17 mRNA compared with those in BMM (Supplemental Figure 1A) (34), and only GM-BMM secreted CCL17 (Supplemental Figure 1B), but again IL-1 $\beta$ and TNF were not detected (data not shown). For ex vivo resident and thioglycolate-elicited peritoneal macrophages, GM-CSF again strongly upregulated Ccl17 mRNA (Supplemental Figure 1C and Figure 1F, respectively); a comparison for the latter macrophage population for Il1b and Tnf mRNA is shown (Figure 1F).

Thus CCL17 is highly secreted in both human and murine monocyte/macrophage populations following GM-CSF addition.

Dependence of CCL17 expression on GM-CSF in vivo. We next determined whether CCL17 expression in vivo was regulated by GM-CSF in the steady state, upon its administration and during inflammatory reactions. Ccl17 mRNA expression could be detected in the hind footpads of naive WT mice, but was significantly lower in the footpads of $\mathrm{GMCSF}^{-/}$mice (Supplemental Figure 2A).

Using CCL17/EGFP reporter ( $\left.C c l 17^{\mathrm{E} /+}\right)(9)$ mice, 4 days following GM-CSF-dependent antigen-induced peritonitis induction (8), CCL17/EGFP expression was detected predominantly in the CD115 (CSF-1R) $)^{+}$inflammatory moDC and macrophage populations (8) (Supplemental Figure 2E). There was also some expression in the minor conventional DC ( $\mathrm{CDC}$ ) population, but not in neutrophils, eosinophils (Supplemental Figure 2E), or lymphocytes (data not shown). Treatment of WT mice with a neutralizing anti-GM-CSF $\mathrm{mAb}$ (days 1 and 2) following antigen-induced peritonitis induction (8) led to a significant reduction in Ccl17 mRNA expression in total peritoneal exudate cells (Supplemental Figure 2F); however, there was no change in Tnf mRNA expression.

These data indicated that GM-CSF upregulates CCL17 expression in the steady state, upon its administration and during inflammatory reactions.

CCL17 is required for GM-CSF-driven inflammatory pain and can itself induce pain. GM-CSF has been shown to induce mechanical hyperalgesia (2) and to be required for the development of inflammatory and arthritic pain $(1,3)$. We therefore examined whether pain driven by GM-CSF administration was dependent on CCL17. GM-CSF was again injected intraplantarly and pain monitored as before by a change in weight distribution (using an incapacitance meter) $(4,5)$. The i.pl. GM-CSF induced pain in a cyclooxygenase-dependent manner, since it was suppressed by the cyclooxygenase inhibitor, indomethacin (Figure 2A). Such GM-CSF-driven pain was also dependent on CCL17, as Ccl1 ${ }^{E / E}$ mice (9) were resistant (Figure 2B) even though Illb and $T n f$ mRNA were similarly increased in the footpads of both 
A

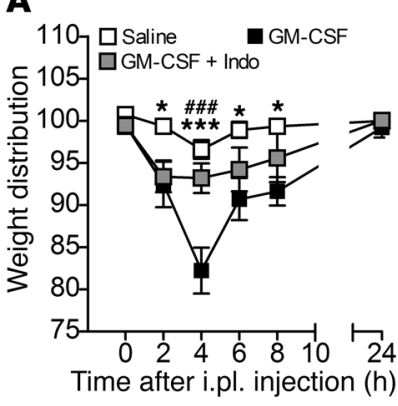

D

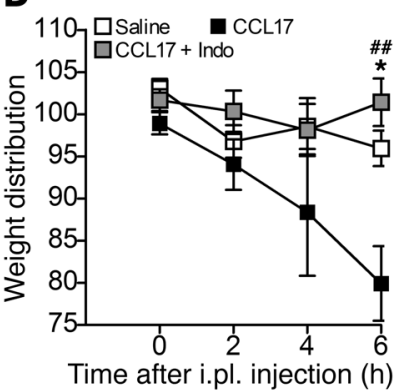

B

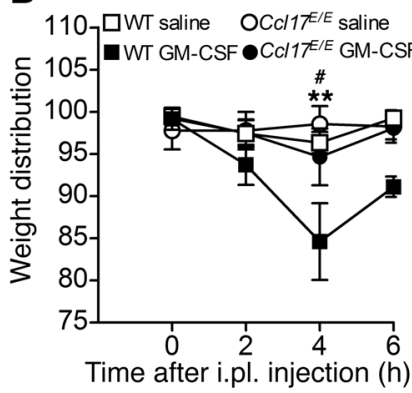

E

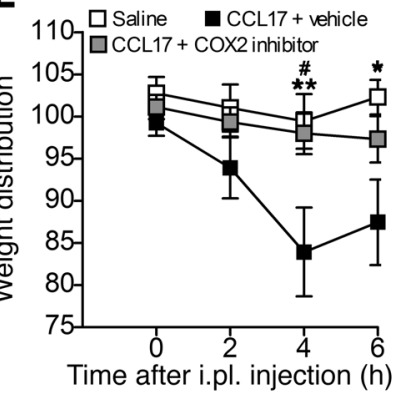

C

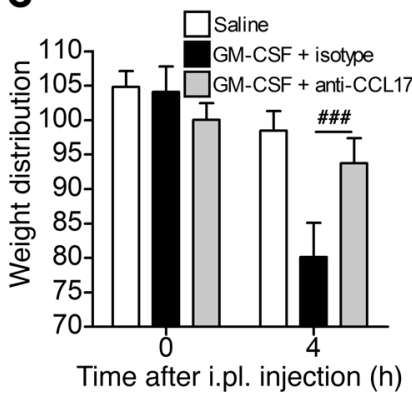

$\mathbf{F}$

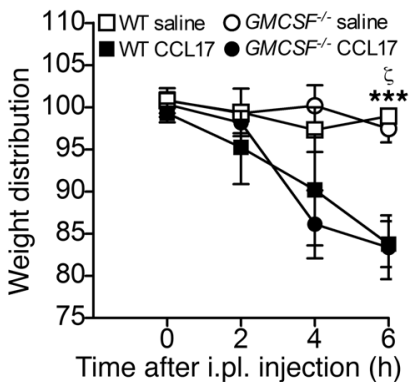

ease (Figure 4C), again consistent with GM-CSF expression not being downstream of CCL17.

The requirement for CCL17 in GM-CSF-dependent inflammatory pain and in GM-CSF-dependent arthritic pain and disease. We have previously found that inflammatory pain, but not footpad swelling (callipers), induced by i.pl. CFA was GM-CSF dependent (3); similarly, using $C c l 17^{E / E}$ mice, the CCL17 dependence of pain in this model, but not swelling (thickness), was demonstrated (Supplemental Figure 3, A and B, respectively). The i.pl. zymosan injection also induces pain (36), which was abrogated using the COX2-specific inhibitor SC58125 (Supplemental Figure 3C). This pain was both GM-CSF and CCL17 dependent (Figure 5, A and C, respectively), but again, the footpad swelling was not (Supplemental Figure 3, D and E, respectively); GMCSF and Ccl17 mRNA were induced in the inflamed paws of WT mice upon zymosan injection (Figure 5, B and D), with the Ccl17 mRNA induction again being GM-CSF dependent (Figure 5B). The increased GMCSF mRNA expression, on the other hand, was not reduced in the zymosan-injected $\mathrm{Ccl1}^{\mathrm{E} / \mathrm{E}}$ mice

$C c l 17^{E / E}$ and WT mice following i.pl. GM-CSF injection (data not shown). Furthermore, the GM-CSF-driven pain was blocked by an anti-CCL17 mAb (Figure 2C).

The i.pl. injection of CCL17 itself led to pain that was also COX dependent (Figure 2D); more specifically, the CCL17-driven pain was dependent on COX2 (Figure 2E). CCL17-driven pain did not require endogenous GM-CSF (Figure 2F), consistent with GM-CSF expression not being downstream of CCL17.

CCL17 is required for GM-CSF-driven arthritic pain and disease and can itself drive arthritic pain and disease. To assess whether CCL17 was also required for GM-CSF-driven arthritic pain and disease, we again took advantage of our mBSA/GM-CSF arthritis model (see Supplemental Figure 2D) (3). Ccl17 ${ }^{\mathrm{E} / \mathrm{E}}$ mice were resistant to $\mathrm{mBSA} / \mathrm{GM}$-CSF-induced arthritic pain and disease (Figure $3 \mathrm{~A}$ ), and anti-CCL17 mAb was able to ameliorate both (Figure 3B).

The substitution of s.c. CCL17 for GM-CSF showed that CCL17 could also exacerbate the mild synovitis induced by i.a. mBSA injection, resulting in pain and disease (Figure 4A). As for pain induction in the mBSA/GM-CSF model (3), indomethacin treatment from day 5 was able to reverse pain within 1 day (Figure $4 \mathrm{~A}$ ), with a trend for reduced disease (Figure $4 \mathrm{~A}$ ). Like the mBSA/GM-CSF model (data not shown), the new mBSA/CCL17 model was as active in $\mathrm{Rag1}^{-/}$mice, indicating $\mathrm{T} / \mathrm{B}$ cell independence (Figure $4 \mathrm{~B}$ ), and did not require GM-CSF for pain or dis-
(Figure 5D), consistent once more with CCL17 not driving GM-CSF expression. Illb and Tnf mRNA were also induced following i.pl. zymosan injection and were significantly lower in $\mathrm{GMCSF}^{-1}$ mice, but not in $\mathrm{Ccl1}^{\mathrm{E} / \mathrm{E}}$ mice (Figure 5, B and D, respectively).

The i.a. zymosan also induces an acute, innate immune-driven monoarticular arthritis (37). Treatment with indomethacin once pain was evident (day 1) led to a reversal of the pain within 1 day, indicating cyclooxygenase dependence (Supplemental Figure $3 F)$. Zymosan-induced arthritic pain was dramatically reduced in $\mathrm{GMCSF}^{2-}$ mice and the arthritis severity (day 7) lowered (Figure 6A). We also found that $\mathrm{Ccl17}$ mRNA expression in zymosan-induced arthritic joints was abolished in GMCSF$^{-1}$ - mice (Figure 6B); Illb and $\operatorname{Tnf}$ mRNA expression were also reduced in the inflamed joints of $\mathrm{GMCSF}^{2}$ - mice compared with WT mice. As for the $\mathrm{GMCSF}^{2-}$ mice, zymosan-induced arthritic pain was essentially absent and the disease severity reduced (Figure 6C) in $\mathrm{Ccl1}^{\mathrm{E} / \mathrm{E}}$ mice.

We have previously shown arthritic pain and disease in the $\mathrm{T}$ cell-dependent antigen-induced arthritis (AIA) model to be dependent on GM-CSF (3); likewise, $\mathrm{Ccl1}^{\mathrm{E} / \mathrm{E}}$ mice did not develop pain following AIA induction and showed significantly less joint destruction (Figure 6D).

The data above in the GM-CSF-driven and GM-CSFdependent models indicate a key role of CCL17 in inflammatory pain and arthritis. 
A
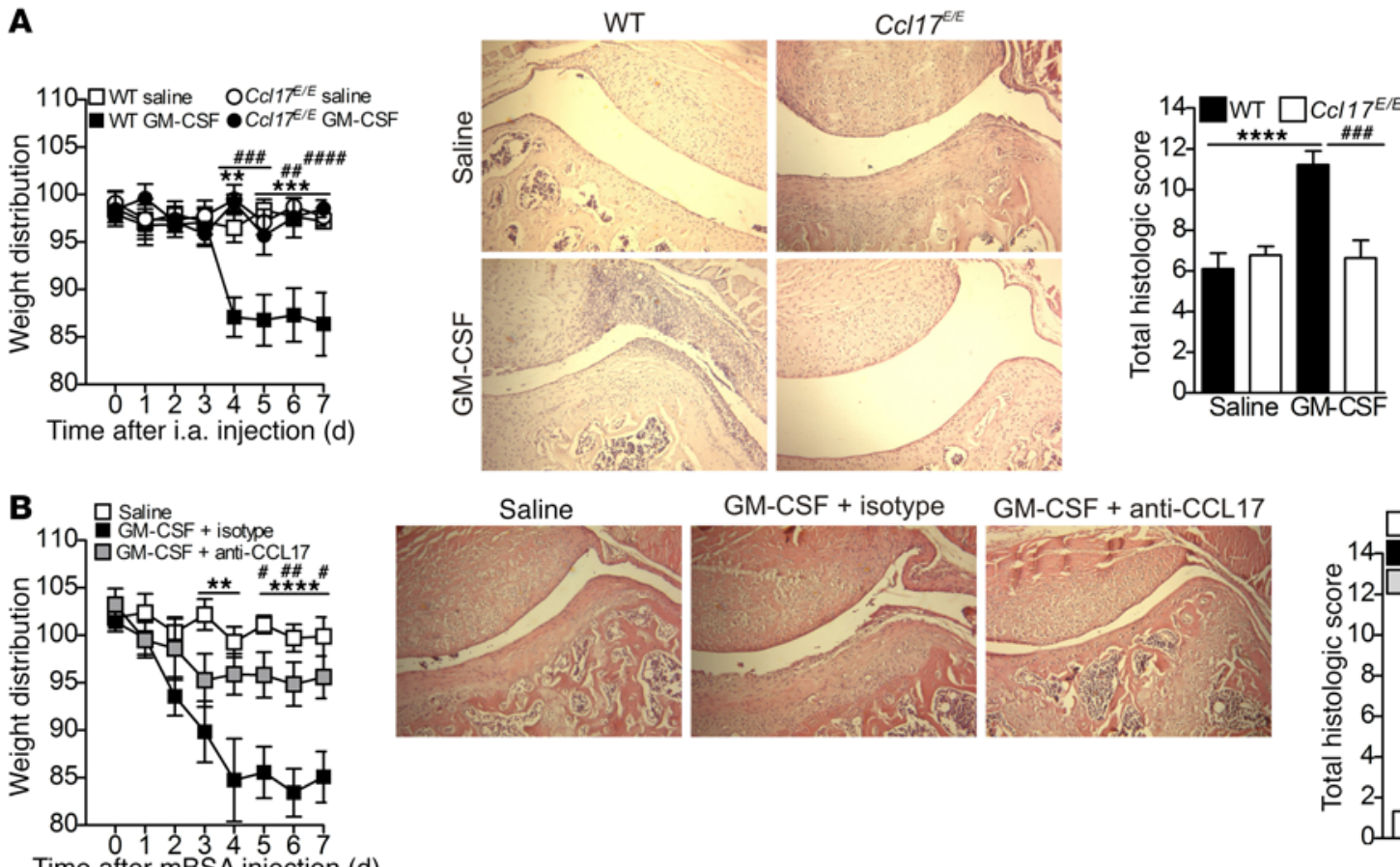

Time after mBSA injection (d)

Figure 3. CCL17 is required for GM-CSF-driven arthritic pain and disease. (A and B) mBSA/GM-CSF arthritis (mBSA i.a.) (day 0) and GM-CSF or saline (days 0 to 2) was induced in (A) WT and Cc/17 $7^{E / E}$ mice ( $n=10$ per group) and (B) WT mice treated with anti-CCL17 or isotype control (150 $\mu \mathrm{g}$ i.p., days -2 and 0 ) ( $n=10$ per group). Pain (incapacitance meter) and arthritis (histology) were measured. Original magnification, $x 125$. Results are shown mean \pm SEM. $P$ values were obtained using a 2-way ANOVA test for pain (weight distribution) readings and a 2-way (A) or 1-way (B) ANOVA test for histology quanti-

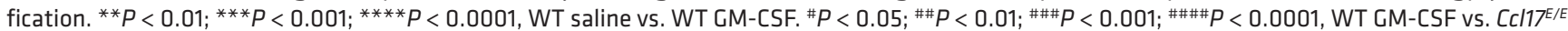
CM-CSF; GM-CSF + isotype vs. GM-CSF + anti-CCL17 mAb.

GM-CSF-induced CCL17 expression in monocytes/macrophages is IRF4 dependent. We next explored the molecular pathway(s) utilized by GM-CSF to upregulate CCL17 expression. We have previously shown that GM-CSF could induce IRF4 expression in human monocytes (31). Although IRF5 has been implicated in the development of a GM-CSF-driven proinflammatory (M1) macrophage phenotype (30), when compared with vehicle (PBS) control, we could detect by microarray a significant increase in only the IRF4 gene among IRF family transcription factors following 16 hours of GM-CSF treatment (data not shown); this was confirmed for IRF4 and IRF5 for mRNA (Figure 7A) and protein (Figure $7 \mathrm{~B}$ ). Silencing the $I R F 4$ gene in human monocytes significantly decreased GM-CSF-induced CCL17 mRNA and secreted protein (Figure 7, C and D, respectively), but not TNF mRNA (Figure 7C); silencing the IRF5 gene had no effect on the expression of either of these mediators.

GM-CSF treatment of M-CSF-starved (CSF-1-starved) BMM resulted in a dramatic induction of Irf4 mRNA (Figure 7E) and protein (Figure 7F), again correlating with CCL17 expression (Figure 1, D and E). In addition, using BMM from $C \mathrm{Cl} 17^{\mathrm{E} /+}$ mice, when

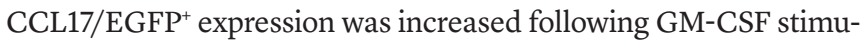
lation, the macrophages also became positive for IRF4 (Figure 7G). GM-CSF-treated BMM from Irf4 ${ }^{-/-}$mice had lower Ccl17 mRNA than GM-CSF-treated WT BMM and no secreted CCL17 (Figure 7, $\mathrm{H}$ and I, respectively). Irf4 deficiency resulted, in contrast, in upregulation of $I l 1 b$ mRNA, but no change in Tnf mRNA (Figure 7H). The GM-CSF-dependent upregulation of Irf4 mRNA expression was also noted in both thioglycolate-elicited (Figure 7J) and resident
(Supplemental Figure 4A) peritoneal macrophages. Ccl17 mRNA expression was significantly lower in both GM-CSF-treated thioglycolate-elicited (Figure 7K) and resident (Supplemental Figure 4B) peritoneal macrophages from Irf $4^{-/}$mice compared with WT mice; Il1 b and Tnf upregulation in GM-CSF-treated Irf4 $4^{-/-}$thioglycolate-elicited macrophages (Figure 7K), however, occurred normally.

GM-BMM had increased levels of Irf4 mRNA and protein compared with BMM (Supplemental Figure 4, C and D, respectively) correlating with CCL17 expression (Supplemental Figure 1, A and B). Using the CCL17/EGFP reporter mice $\left(C c l 17^{E /+}\right)$, a higher proportion of GM-BMM were CCL17/EGFP ${ }^{+}$compared with BMM (Supplemental Figure 4D) and the amount of IRF4 protein expressed by

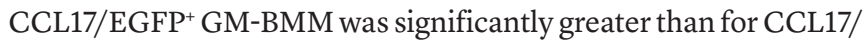
$\mathrm{EGFP}^{-} \mathrm{GM}-\mathrm{BMM}$ (mean fluorescence intensity [MFI] $8.9 \pm 0.4 \mathrm{vs}$. $1.4 \pm 0.1, P<0.0001)$. Withdrawal of GM-CSF from GM-BMM cultures resulted in a decrease in IRF4 protein (Supplemental Figure 4E). GM-BMM from Irf4 $4^{--}$mice had significantly lower levels of Ccl17 mRNA, an upregulation of Il1b mRNA, and a trend toward an increase in Tnf mRNA compared with WT GM-BMM (Supplemental Figure $4 \mathrm{~F}$ ), which was similar to that of Irf4 ${ }^{-/}$BMM treated with GM-CSF (Figure 7H). Paralleling the mRNA data, secreted CCL17 protein was markedly lower in GM-BMM from Irf4 $4^{-/-}$mice (Supplemental Figure 4G). IRF4 expression would not appear to be dependent on endogenous CCL17 in the GM-BMM cultures, since its mRNA and protein levels were equivalent between WT GM-BMM and Ccl17 ${ }^{E / E}$ GM-BMM (Supplemental Figure 5, A and $\mathrm{B}$, respectively). Thus, the CCL17 induction by GM-CSF in monocytes/macrophages is IRF4 dependent. 
A

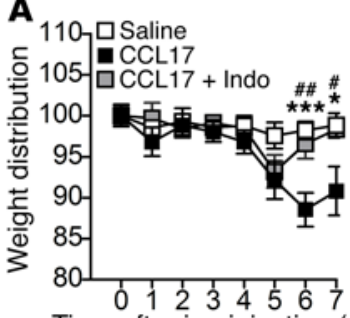

Time after i.a. injection (d)

B

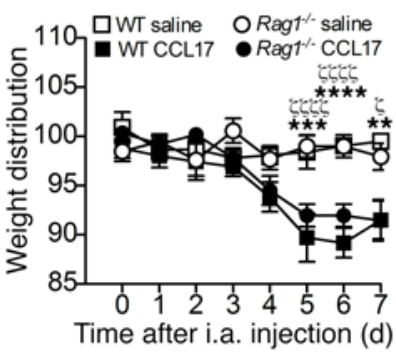

C

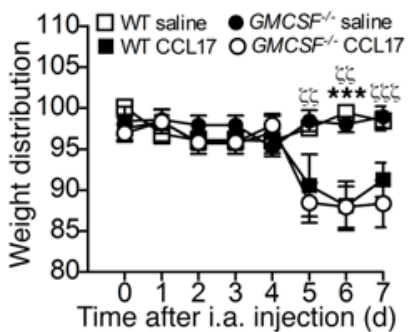

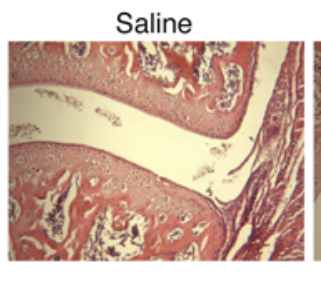
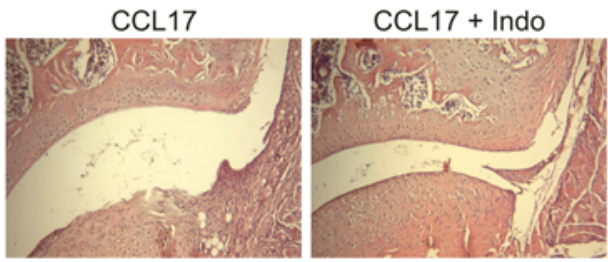

WT

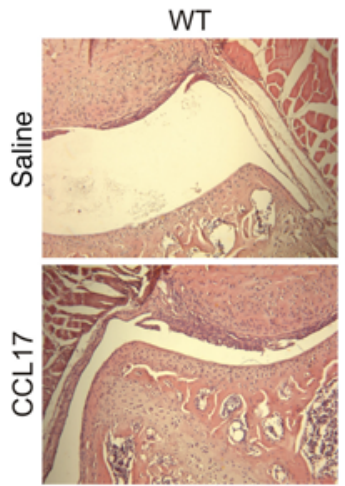

WT

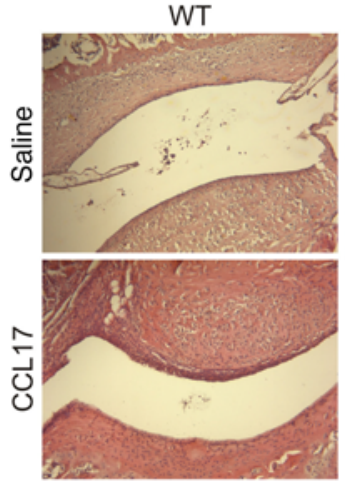

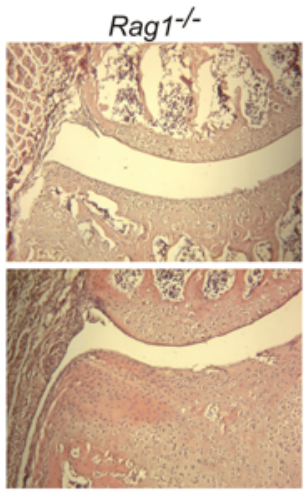

GMCSF-

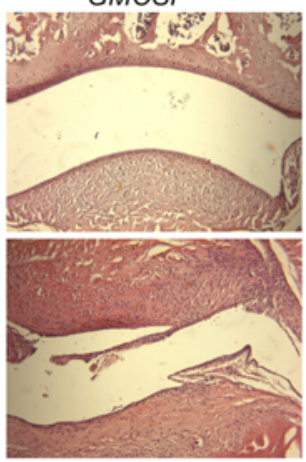

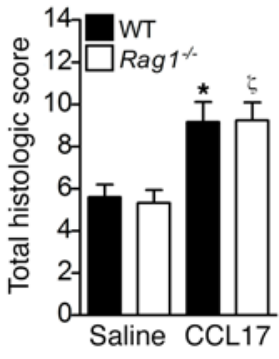

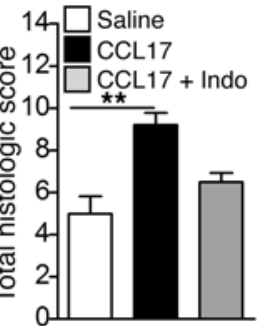

Figure 4. CCL17 can drive arthritic pain and disease. (A-C) mBSA/CCL17 arthritis (mBSA i.a. [day 0 ] and CCL17 or saline [days 0 to 2]) was induced in (A) WT mice treated with indomethacin ( $1 \mathrm{mg} / \mathrm{kg}$ i.p.) or PBS from day 5 ( $n=5$ per group), (B) WT and Rag $1^{-/-}$mice $\left(n=6\right.$ per group), and (C) WT and CMCSF ${ }^{-/-}$ mice ( $n=6$ per group). Pain (incapacitance meter) and arthritis (histology) were measured. Original magnification, $\times 125$. Results are shown as mean \pm SEM. $P$ values were obtained using a 2-way ANOVA test for pain (weight distribution) readings and a 1-way (A) or 2-way (B and C) ANOVA test for histology

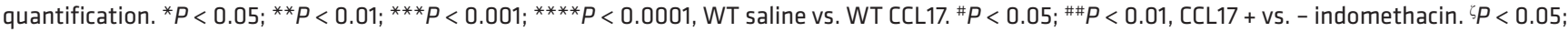

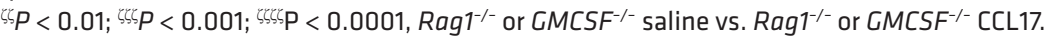

IRF4 is required for GM-CSF-driven inflammatory pain and for GM-CSF-driven arthritic pain and disease. If IRF4 is also involved in the GM-CSF-dependent upregulation of CCL17 in vivo, we predicted that our GM-CSF-driven inflammatory pain and arthritis models would also be IRF4 dependent. The i.pl. injection of GM-CSF in Irf4 ${ }^{-/}$mice resulted in no pain (Figure 8A) and no increased Ccl17 mRNA expression, whereas the increases in Illb and Tnf mRNA were not affected (Figure 8B); in contrast, i.pl. injection of CCL17 in Irf $4^{-/}$mice led to a level of pain similar to that observed in WT mice (Figure 8C).

In the i.a. mBSA-injected (arthritic) joints of WT mice undergoing the mBSA/GM-CSF arthritis protocol, Irf4 mRNA expression was increased (Figure 8D); this model required IRF4 for both pain and disease (Figure 8E). In this model, Ccl17 mRNA was lower in the mBSA-injected (arthritic) joints of Irf4- mice compared with WT mice (Figure 8F), and the same was evident for $I l 1 b$ and Tnf mRNA (data not shown), possibly due to reduced joint inflammation over the 7-day period (Figure 8E). In contrast, the mBSA/CCL17 arthritis model did not require IRF4 (Figure $8 \mathrm{G}$ ). These data support the in vivo relevance of the proposed new GM-CSF/IRF4/CCL17 pathway.

$I R F 4$ is required for GM-CSF-dependent inflammatory and arthritic pain. We also predicted that GM-CSF-dependent inflammatory and arthritic pain would require IRF4. Irf4 ${ }^{-/}$mice developed less pain than WT mice, but did develop footpad swelling following i.pl. zymosan injection (Figure 9, A and B, respectively). Also, footpad Ccl17 mRNA expression in Irf4-/ mice did not increase following i.pl. zymosan injection, unlike in WT mice (Figure 9C); however, there were no differences in the increased mRNA expression of GMCSF, Illb, and Tnf between WT and Irf $4^{-/}$mice (Figure 9C). Zymosan-induced arthritic pain and disease were also IRF4 dependent (Figure 9D). Using Rag1 ${ }^{--}$mice, we found zymosan-induced arthritic pain and disease to be T/B cell independent (data not shown). These data again support the in vivo relevance of the proposed new GM-CSF/IRF4/CCL17 pathway. 


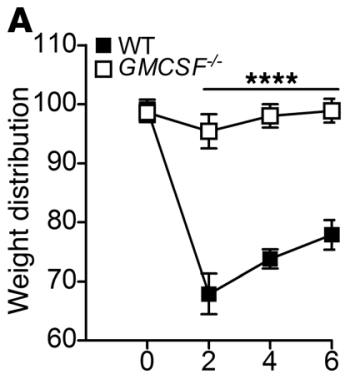

Time after i.pl. injection (h)

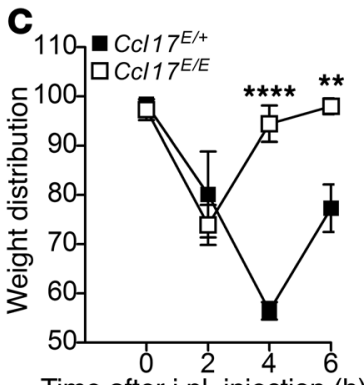

Time after i.pl. injection (h)
B

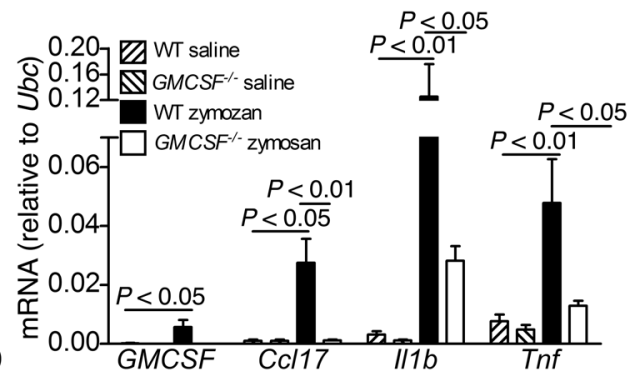

D

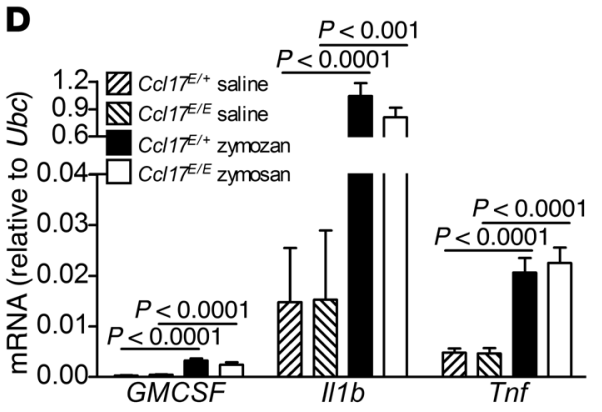

Figure 5. The requirement for CCL17 in GM-CSF-dependent inflammatory pain. (A and $\mathbf{B}) \mathrm{WT}$ and $\mathrm{CMCSF}^{-/-}$mice received an i.pl. injection of zymosan, and (A) pain (incapacitance meter) and (B) footpad mRNA expression (qPCR) (6 hours zymosan or saline) were measured ( $n=10$ per group). (C and $\mathbf{D}$ ) Cc/17 ${ }^{E /+}$ and $C c / 17^{E / E}$ mice received an i.pl. injection of zymosan, and (C) pain and (D) footpad mRNA expression (6 hours zymosan or saline) were measured ( $n=7-8$ per group). Results are shown as mean \pm SEM. $P$ values were obtained using 2-way ANOVA tests. ${ }^{* *} P<0.01 ;{ }^{* * *} P<0.0001, \mathrm{WT}$ or $\left[c / 17^{E /+}\right.$ vs. $\mathrm{CMCSF}^{-/-}$or $\left[\mathrm{C} / 17^{\mathrm{E} / \mathrm{E}}\right.$ mice.
GM-CSF regulates IRF4 expression via JMJD3 demethylase in human monocytes. The promoter region of IRF4 is enriched in trimethylated histone H3Lys27 (H3K27me3), which can be converted by JMJD3 (KDM6B) demethylase to its monomethylated state, thereby enhancing its transcription (38). We therefore hypothesized that GM-CSF might regulate JMJD3 expression and/or activity, thereby controlling IRF4 transcription. GM-CSF treatment of human monocytes increased KDM6B mRNA, with its peak occurring before the increased IRF4 and CCL17 transcripts (Figure 10A); JMJD3 activity (Figure 10B) and protein (Figure 10C) were also elevated. Importantly, the increase in IRF4 protein correlated with a dramatic decrease in $\mathrm{H} 3 \mathrm{~K} 27 \mathrm{me} 3$, while total histone 3 expression remained the same (Figure 10C). Treatment with the JMJD3 inhibitor GSK-J4 before GM-CSF stimulation resulted in trimethlyation of $\mathrm{H} 3 \mathrm{~K} 27$ that is comparable to that of unstimulated monocytes while GM-CSF-induced IRF4 protein was decreased (Figure 10C). As hypothesized, the pretreatment had an effect on GM-CSF-regulated JMJD3 activity, leading to a marked decrease in GM-CSF-induced CCL17 (Figure 10D), correlating with the reduction in IRF4. Furthermore, ChIP assays confirmed that GSK-J4 blocked the recruitment of RNA polymerase II to the IRF4 transcription start site (TSS) and also prevented the GM-CSF-induced loss of $\mathrm{H} 3 \mathrm{~K} 27 \mathrm{me} 3$ association to this locus, while not altering the total $\mathrm{H} 3$ at this site (Figure 10E).

The JMJD3 inhibitor GSK-J4 inhibits GM-CSF-driven inflammatory pain. To assess whether blockade of JMJD3 activity would ameliorate GM-CSF-driven pain development, WT mice were pretreated i.p. with GSK-J4 or vehicle 30 minutes prior to i.pl. GM-CSF. Mice pretreated with vehicle prior to GM-CSF developed pain, whereas mice treated with the JMJD3 inhibitor were resistant to pain development (Figure 10F). This finding suggests that development of GM-CSF-driven pain requires JMJD3 activity.

\section{Discussion}

We have identified a new GM-CSF-driven pathway in monocytes/ macrophages leading to CCL17 formation and with upstream involvement of JMJD3-regulated IRF4; this pathway was identified in both human and murine cells in vitro, and also in vivo, in the steady state and also during inflammatory responses. This pathway appears to be separate from the GM-CSF-driven pathway(s) in monocytes/macrophages, leading to expression of other key proinflammatory cytokines, such as IL-1 $\beta$ and TNF. We also found that CCL17 was secreted from all the GM-CSF-treated monocyte/macrophage populations tested, unlike, for example, IL-1 $\beta$ and TNF, which could not be detected and which usually require an additional stimulus, such as LPS $(32,33,39)$. By using both GM-CSFdriven and GM-CSF-dependent models, we have demonstrated that CCL17 can mediate at least some of the proinflammatory and algesic actions of GM-CSF. Consistent with our data, Ccl17 mRNA is strikingly reduced in inflammatory moDCs lacking functional GM-CSF receptors (40), although it cannot be assumed that the new pathway is operating exclusively in monocytes/macrophages/ DCs during inflammation. Our data are the first, to our knowledge, to indicate a role for CCL17 in the propagation of inflammatory pain as well as in arthritic pain and disease. What other downstream mediators are formed via this pathway remains to be determined. From our findings, GM-CSF joins the list of stimuli, for example, IL-4 (41, 42), IL-13 (43), and thymic stromal lymphopoietin (TSLP) (44), associated with upregulation of CCL17, which has traditionally been viewed as a chemokine participating in Th2-type immune responses $(9,10)$, leading to it usually being considered as a marker of M2 macrophage polarization $(45,46)$.

CCL17 has been reported to be required for CCR7- and CXCR4-dependent migration of dermal DCs, suggesting a wider chemotactic role (12). The data above showing that CCL17 has a nonredundant function in models that are $\mathrm{T} / \mathrm{B}$ cell independent indicate that CCL17 is not acting as a T cell chemokine in these models. The COX2 dependence of the CCL17-driven inflammatory and arthritic pain also suggests that CCL17 may have other roles in inflammation in addition to being a chemokine $(13,16)$.

Several pieces of evidence point toward IRF4 being a key intermediary in the GM-CSF-driven upregulation of CCL17 formation 

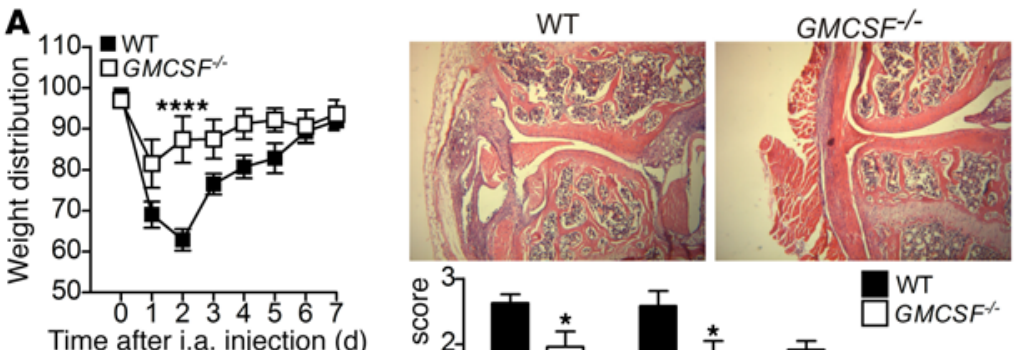

옹 ${ }^{3}$

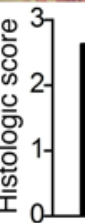

WT

GMCSF

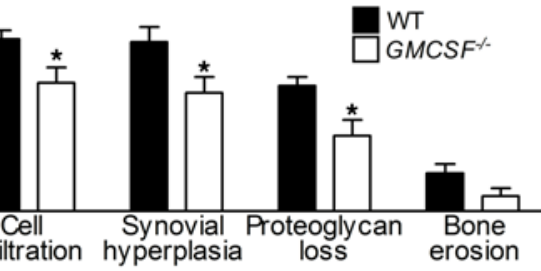

$\mathrm{Ccl} 17^{\mathrm{E}}$

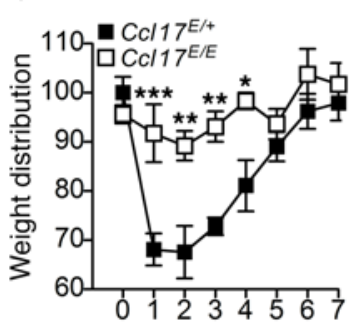

Time after i.a. injection (d)
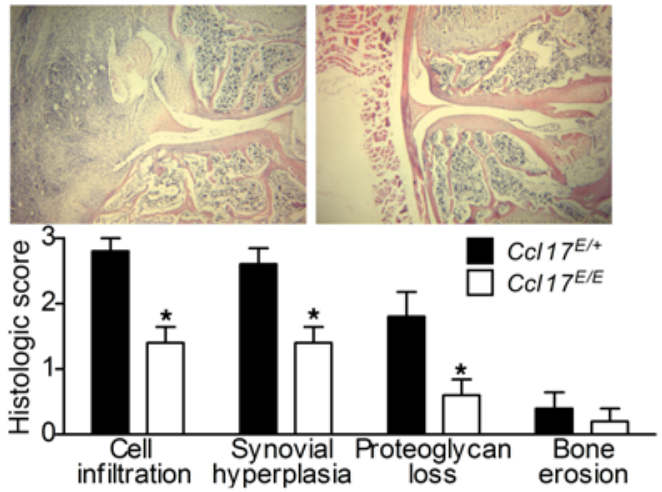

WT
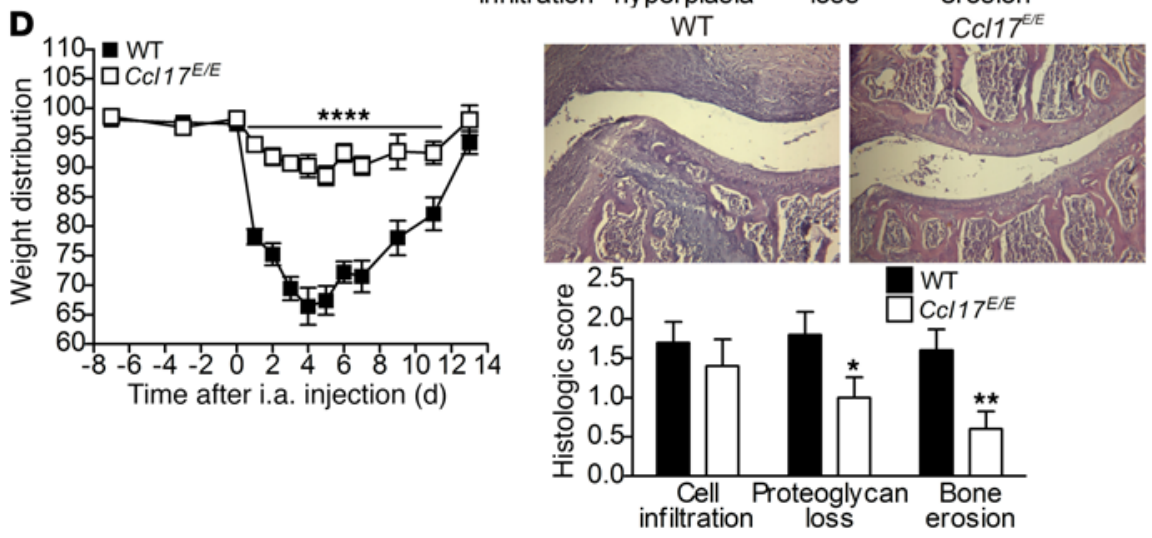

B

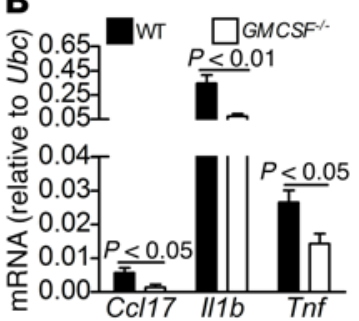

Figure 6. The requirement for CCL17 in GM-CSF-dependent arthritic pain and disease. (A and B) WT and GMCSF ${ }^{-/-}$mice received an i.a. injection of zymosan, and (A) pain and arthritis (histology, day 7) and (B) joint mRNA expression (day 7) were measured $\left(n=10\right.$ per group). (C) $C \mathrm{C} / 17^{E /+}$ and $C \mathrm{C} / 17^{E / E}$ mice received an i.a. injection of zymosan, and pain and arthritis (histology, day 7) were measured ( $n=5$ per group). (D) Pain and arthritis (histology, day 14) development in WT and Cc/17 E/E mice with AIA ( $n=15$ per group). Results are shown as mean \pm SEM. $P$ values were obtained using a 2-way ANOVA test $(\mathbf{A}, \mathbf{C}$, and $\mathbf{D})$ for pain readings, Mann-Whitney $U$ test (A, C, and $\mathbf{D})$ for histology, and a $t$ test (B) for gene expression. ${ }^{*} P<0.05 ;{ }^{* *} P<0.01 ;{ }^{* *} P<0.001$; ${ }^{* * * *} P<0.0001$, WT or CC/17E/+ vs. CMCSF ${ }^{-/-}$or CC/17 $7^{E / E}$ mice. Original magnification, $\times 60$.

and in GM-CSF biology in general. Our in vivo experiments examining the IRF4 contribution used only models for which mature lymphocytes were not required in order to reduce any complexities arising from the known IRF4 regulation of lymphocyte development (21). The effects of IRF4 depletion on GM-CSF-driven and GM-CSF-dependent pain, swelling, and arthritis were similar to those observed upon CCL17 deletion/depletion, supporting our pathway. Our data also indicate that IRF4 may have a proinflammatory role in monocytes/macrophages in contrast with the literature in which it has been considered to have an antiinflammatory function in macrophages in vitro $(26,27)$ and in vivo $(26,28)$. The heterogeneity of the GM-CSF-derived cells from murine bone marrow cells, often used as a model for DCs and/or macrophages (termed GM-BMM above and elsewhere; refs. 33, 34), has been highlighted $(25,47,48)$, with an IRF $4^{+}$population being defined as a relatively mature PDL2 $2^{+} \mathrm{DC}(25,48)$. We suggest that this is present in our GM-BMM cultures as the IRF $4{ }^{+} \mathrm{CCL} 17^{+}$population (Supplemental Figure 4D).

Our prior data (31) and also data presented here point toward IRF4, rather than IRF5 (30), as being more critical for the action of GM-CSF on monocytes/macrophages. IRF5 has been proposed to be important for GM-CSF-driven M1 (proinflammatory) macrophage polarization (30), while IRF4 has been linked to M2 macrophage polarization, with an upstream JMJD3-mediated 

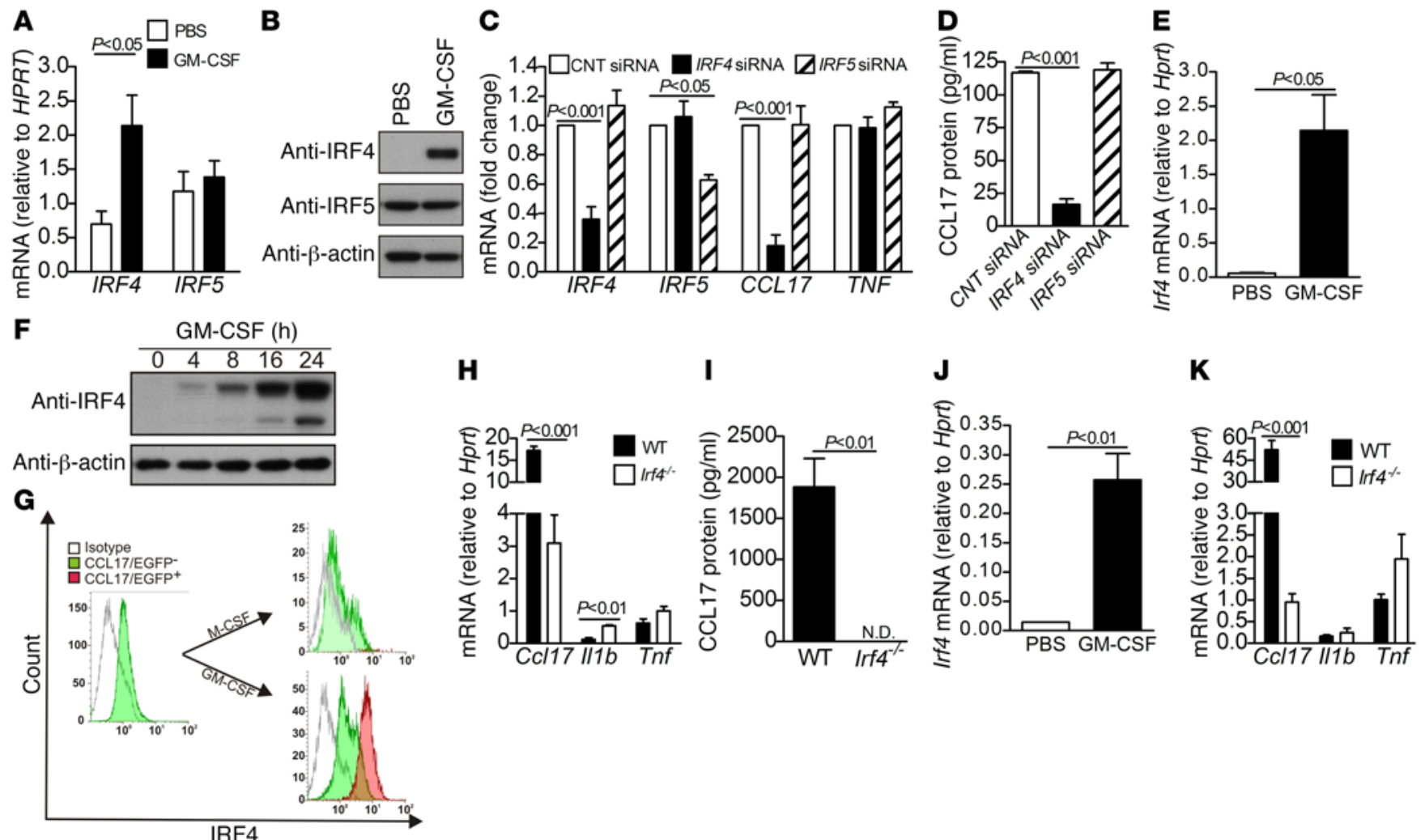

Figure 7. GM-CSF-induced CCL17 expression in monocytes/macrophages is IRF4 dependent. (A and B) Human monocytes were treated with either PBS or CM-CSF (10 ng/ml) for 16 hours. (A) IRF4 and IRF5 mRNA expression (qPCR) and (B) whole cell lysates were subjected to Western blotting with anti-IRF4, anti-IRF5, and anti- $\beta$-actin antibodies $(n=4)$. (C and D) Human monocytes were nucleofected with IRF4, IRF5, or a control nontargeting (CNT) siRNA before being stimulated with GM-CSF $(10 \mathrm{ng} / \mathrm{ml})$ for 16 hours. (C) IRF and cytokine/chemokine mRNA expression plotted relative to that in CNT siRNA, which was given an arbitrary value of 1.0, and (D) secreted CCL17 by ELISA $(n=5)$. (E and F) Murine bone marrow cells were cultured in M-CSF $(5,000 \mathrm{U} /$ $\mathrm{ml}$ ) for 7 days to derive BMM followed by GM-CSF treatment in the absence of M-CSF. (E) Irf4 mRNA expression (24 hours) and (F) whole cell lysates from BMM treated with GM-CSF for the indicated periods of time were subjected to Western blotting with anti-IRF4 and anti- $\beta$-actin antibodies $(n=4)$. (C) Representative histograms of IRF4 in CCL17/EGFP+ and CCL17/EGFP- populations of BMM (from Cc/17//+ mice) treated with fresh medium containing either M-CSF or GM-CSF for 72 hours $(n=3)$. (H and I) BMM from WT or Irf4 $4^{-/-}$mice were stimulated with GM-CSF $(10 \mathrm{ng} / \mathrm{ml})$ for 24 hours in the absence of M-CSF. (H) Cytokine mRNA expression and (I) secreted CCL17 protein in the supernatant (ELISA) $(n=4)$. (J and K) Thioglycolate-elicited peritoneal macrophages were treated with PBS (vehicle) and GM-CSF $(20 \mathrm{ng} / \mathrm{ml})$ for 24 hours. (J) Irf4 mRNA expression in WT macrophages and (K) cytokine mRNA expression in WT and Irf4 ${ }^{-/}$macrophages $(n=4)$. Graphs are plotted as mean \pm SEM. $P$ values were obtained using a $t$ test.

demethylation being crucial $(38,49)$; however, GM-CSF has also been viewed as an M2 cytokine (see, for example, ref. 50). We have provided evidence that JMJD3 is important in the GM-CSF-stimulated upregulation of IRF4 and ultimately CCL17, which is the first demonstration, to our knowledge, that such an epigenetic mechanism can contribute to GM-CSF signaling. Importantly, a JMJD3 inhibitor ameliorated GM-CSF-driven inflammatory pain. JMJD3 expression has been reported to be controlled by NF- $\mathrm{BB}$ in LPSactivated BMM (51). We previously showed that, following LPS stimulation, GM-BMM displayed increased NF- $\mathrm{kB}$ activity compared with BMM (33), suggesting that GM-CSF could potentially control JMJD3 expression via NF-kB. While it remains unknown how GM-CSF might regulate IRF4 expression in monocytes/ macrophages, a recent study found that STAT5 could bind to the IRF4 promoter in $\mathrm{T}$ cells, thus regulating its expression (52). Given that GM-CSF activates the JAK2/STAT5 signaling pathway (6), it is tempting to speculate that it may regulate IRF4 expression via STAT5 in monocytes/macrophages as well. Our data also reinforce the recommendation that the $\mathrm{M} 1 / \mathrm{M} 2$ nomenclature should not be used to describe GM-CSF-treated monocytes/macrophages (46).
It is noteworthy that depletion of both CCL17 and IRF4 had very dramatic effects on pain in the various i.pl. and arthritis models without effects on swelling, i.e., pain and swelling were dissociated, which has been reported previously (see, for example, ref. 53). These findings were similar to what we found before upon GM-CSF deletion/depletion (3), again providing supporting evidence for our proposed pathway and for some selectivity in its contribution to an inflammatory response. In addition, GM-CSFand CCL17-driven, as well as GM-CSF- and CCL17-dependent, inflammatory and arthritic pain were all blocked by cyclooxygenase inhibition (3), suggesting that eicosanoids also provide another link between the biology of these 2 cytokines.

Both GM-CSF (54) and CCL17 (55) expression are elevated in rheumatoid arthritis (RA) synovial fluid, with CCL17 expression also elevated in DCs in this fluid (56) - perhaps GM-CSF contributes to this CCL17 expression. Since elevated expression of both GM-CSF (4-6) and CCL17 (13, 16-20) has also been observed in many preclinical and clinical settings, for example, for atopic dermatitis, allergy, lung disease, inflammatory bowel disease, and cardiovascular disease, it could be that the GM-CSF/IRF4/CCL17 


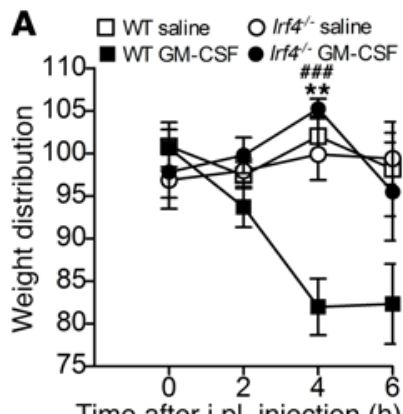

Time after i.pl. injection (h)

E

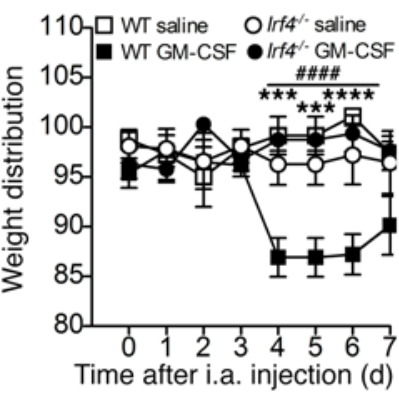

G

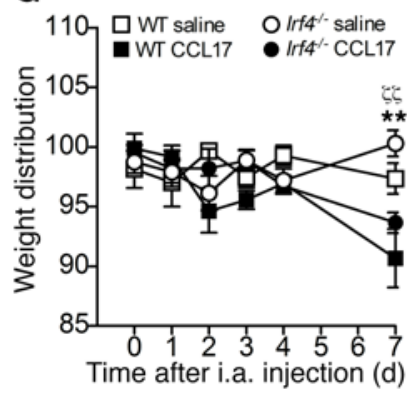

B

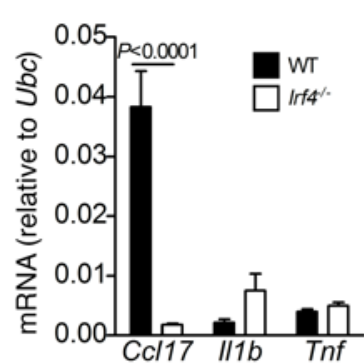

WT

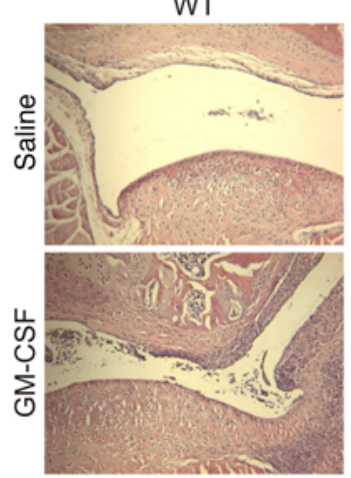

WT

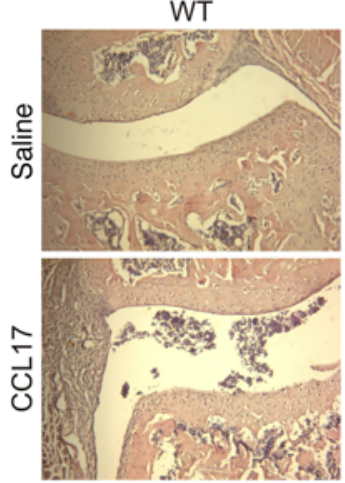

C

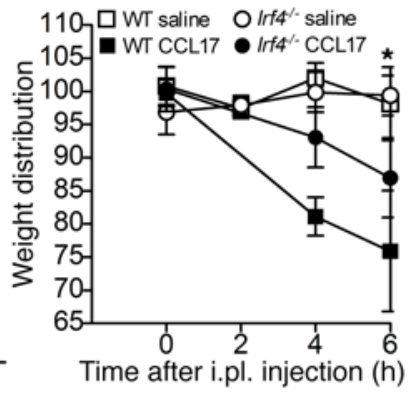
Irf $4^{-/-}$
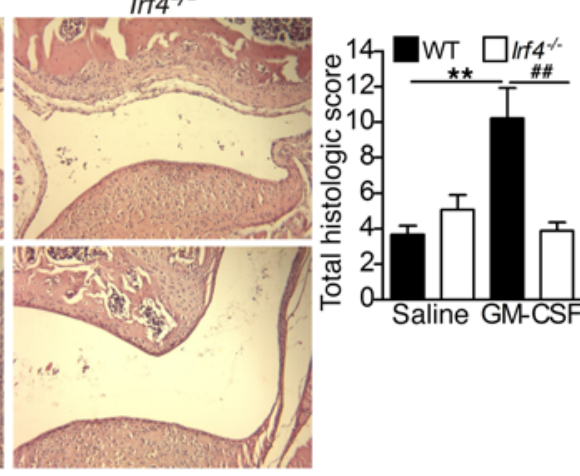

Irf $4^{-/}$

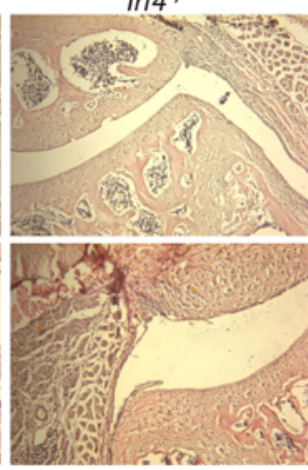

D
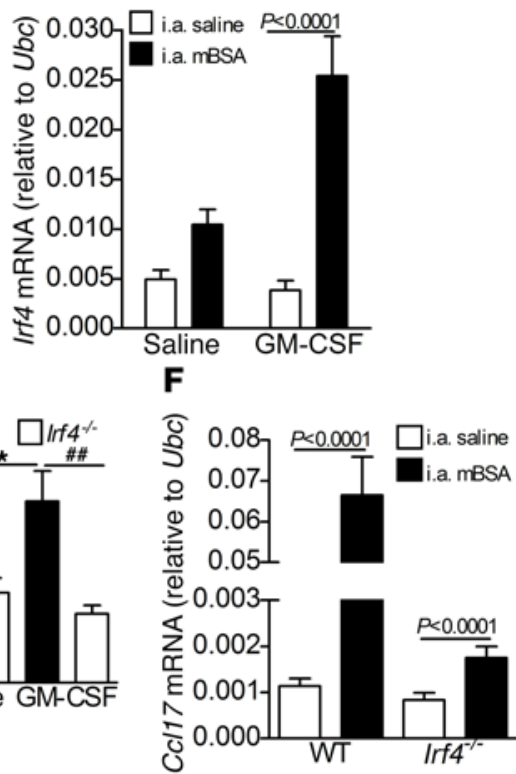

Figure 8. IRF4 is required for GM-CSF-driven inflammatory pain and GM-CSF-driven arthritic pain and disease. (A and B) i.pl. injection of GM-CSF (20 ng) in WT and $/ r f 4^{-/-}$mice. (A) Pain (incapacitance meter) and (B) footpad mRNA expression (qPCR) (4 hours) ( $n=6$ per group). (C) Pain (incapacitance meter) following i.pl. CCL17 (50 ng) in WT and Irf4 ${ }^{-/}$mice ( $n=5$ per group). (D) Irf4 mRNA expression (day 7$)$ in the joints of WT mice with mBSA/GM-CSF arthritis ( $n=6$ per group). (E and F) mBSA/GM-CSF arthritis was induced in WT and Irf4 $4^{-/-}$mice and (E) pain and arthritis (histology, day 7$)$ and (F) joint $C c / 17$ mRNA expression (day 7) were measured ( $n=6$ per group). (C) $\mathrm{mBSA} / \mathrm{CCL} 17$ arthritis model was induced in WT and Irf4 ${ }^{-/}$mice, and pain and arthritis (histology, day 7) were measured ( $n=6$ per group). Original magnification, $x 125$. Results are shown as mean \pm SEM. $P$ values were obtained using a 2 -way ANOVA test $(\mathbf{A}, \mathbf{C}, \mathbf{D}, \mathbf{E}, \mathbf{F}, \mathbf{C})$ and a $t$ test $(\mathbf{B}) .{ }^{*} P<0.05 ;{ }^{* *} P<0.01 ;{ }^{* * *} P<0.001 ;{ }^{* * *} P<0.0001$, WT saline vs. WT GM-CSF or WT CCL17; ${ }^{\# \# P}<0.01{ }^{\# \# P}<0.001$;

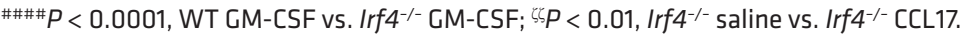

pathway has widespread relevance to pathology and its associated pain. Our findings mean that CCL17 may also be a therapeutic target in conditions where GM-CSF already appears to be one and perhaps without some of the possible side effects, such as pulmonary alveolar proteinosis and infections $(4,5)$.

\section{Methods}

Mice. GMCSF gene-deficient (GMCSF-/) mice, originally provided by the Ludwig Institute for Cancer Research (Parkville, Victoria, Australia), were as previously described $(1,3,8)$. Ccl17 gene-deficient $\left(C c l 17^{E / E}\right)$ and $C c l 17$ heterozygous $\left(C c l 17^{E /+}\right)$ mice, in which either both copies or a single copy of Ccl17 has been replaced by EGFP, are as reported (9). Irf4 ${ }^{-/}$mice were from T.W. Mak (The Campbell Family Institute for Breast Cancer Research, University of Toronto, Toronto,
Canada) (57) and Rag1 ${ }^{-/-}$mice (58) from the Walter and Eliza Hall Institute. Mice were backcrossed onto the C57BL/6 background (Walter and Eliza Hall Institute) for more than 10 generations. Mice of both sexes (8 to 12 weeks) were used.

Isolation of human monocytes. Human monocytes were isolated as described before (31). Briefly, human monocytes were purified from buffy coats (Red Cross Blood Bank, Melbourne, Victoria, Australia), using RosetteSep Ab mixture (Stem Cell Technologies), which negatively select $\mathrm{CD} 14^{+}$monocytes, followed by Ficoll-Paque density gradient centrifugation. They were cultured in RPMI 1640, supplemented with $10 \%$ heat inactivated FCS, 2 mM GlutaMax-1 (Life Technologies), $100 \mathrm{U} / \mathrm{ml}$ penicillin, and $100 \mathrm{mg} / \mathrm{ml}$ streptomycin and treated with either human GM-CSF (10 ng/ml, R\&D Systems) or PBS, for indicated time periods. 

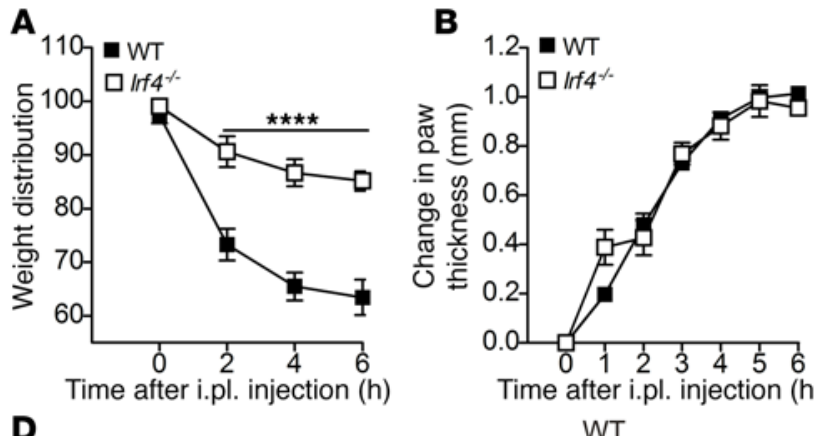

C
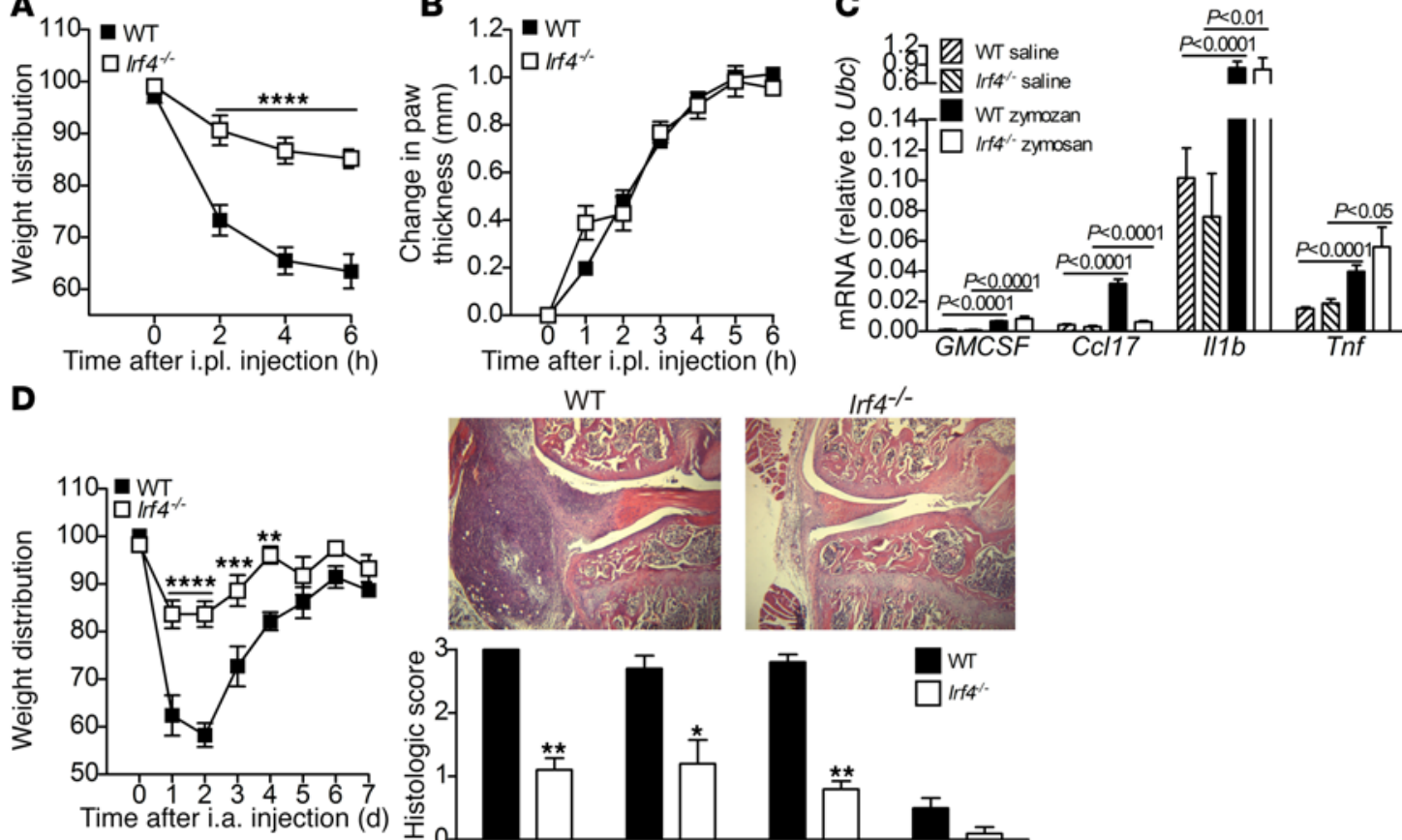
WT

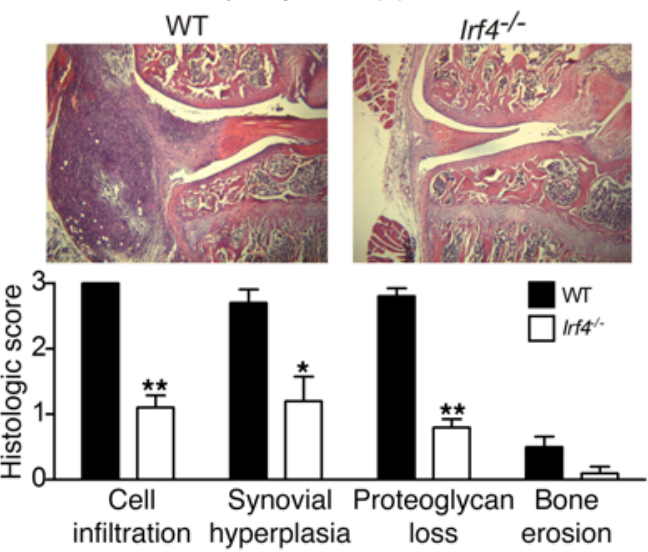

Figure 9. IRF4 is required for GM-CSF-dependent inflammatory and arthritic pain and disease. (A-C) WT and Irf4-/- mice received i.pl. zymosan, and (A) pain, (B) swelling, and (C) mRNA expression (6 hours zymosan or saline) in the footpad were measured ( $n=8$ per group). (D) Pain and arthritis (histology, day 7) development following zymosan-induced arthritis in WT and Irf4-/- mice ( $n=5$ per group). Results are shown as mean \pm SEM. $P$ values were obtained using a 2-way ANOVA test (A-D) for pain and gene expression and Mann-Whitney $U$ test (D) for histology. Original magnification, $\times 60 .{ }^{*} P<0.05$; ${ }^{* *} P<0.01$; ${ }^{* *} P<0.001$; ${ }^{* * *} P<0.0001$, WT vs. Irf4 ${ }^{-1-}$ mice.

Isolation and culture of BMM. BMM and GM-BMM were prepared as previously described (31). Briefly, bone marrow cells were isolated from femurs of mice and cultured in RPMI 1640 medium supplemented with $10 \%$ heat-inactivated FCS, 2 mM GlutaMax-1, $100 \mathrm{U} / \mathrm{ml}$ penicillin, and $100 \mathrm{mg} / \mathrm{ml}$ streptomycin in the presence of either M-CSF $(5,000 \mathrm{U} / \mathrm{ml}$, Chiron) or murine GM-CSF (20 ng/ml, PeproTech). At day 4 , nonadherent cells were collected and cultured for a further 3 days again in either M-CSF $(5,000 \mathrm{U} / \mathrm{ml})$ or $\mathrm{GM}-\mathrm{CSF}(20 \mathrm{ng} / \mathrm{ml})$ to derive BMM and GM-BMM, respectively.

Microarray. Microarray experiments were performed following recommended protocols supplied by Agilent Technologies as before (31). Briefly, human monocytes from 3 independent donors were treated with GM-CSF (10 ng/ml) or PBS for 16 hours before RNA was isolated. RNA integrity was analyzed using an Experion automated electrophoresis station (Bio-Rad). Total RNA ( $1 \mu \mathrm{g})$ was used as starting material, which was amplified using the Low RNA Input Linear Amplification Kit (Agilent Technologies). The labeled cDNA was purified using an RNeasy Mini Kit (QIAGEN) and eluted and quantified using a NanoDrop ND-1000 spectrophotometer (Thermo Fisher Scientific Inc.). Following fragmentation, the cRNA was hybridized to Agilent Whole Human Genome microarray (G4112F) containing 43,376 probes corresponding to 41,264 transcripts. Microarrays were then scanned using a DNA microarray scanner, model G2565A (Agilent Technologies). Intensity data were read using the read. maimages function from the limma Bioconductor R package with background correction using the normexp method (https://www. bioconductor.org/packages/release/bioc/html/limma.html). Since standard methods failed to produce satisfactory results, fold changes and $P$ values were obtained using the RUVinv function from ruv $\mathrm{R}$ package $(59,60)$ (http://www-personal.umich.edu/ johanngb/ruv/ index.html). The list of control genes was from Eisenberg et al. (61). The data set and technical information compliant with minimum information about a microarray experiment (MIAME) (62) can be found at the ArrayExpress Archive Web site (http://www.ebi.ac.uk/ arrayexpress/; E-MTAB-2212).

qPCR. qPCR experiments were performed as described previously (31). Briefly, total RNA was extracted using Isolate II RNA Mini Kit (Bioline) and reverse transcribed using SuperScript III reverse transcriptase (Life Technologies). qPCR was performed using an ABI PRISM 7900HT sequence detection system (Applied Biosystems) and predeveloped TaqMan probe/primer combinations for human and for murine CCL17, IL1B, TNF, IL-23p19, IL-6, GM-CSF, IRF4, IRF5, KDM6B, UBC, and HPRT (Life Technologies). All samples were assayed in duplicate. Threshold cycle numbers were transformed to $\triangle \mathrm{Ct}$ values, and the results were expressed relative to reference genes, UBC or HPRT.

ELISA. Secreted human and mouse CCL17, IL-1 $\beta$, and TNF (R\&D Systems) were measured by ELISA as per the manufacturer's instructions.

Antigen-induced peritonitis. Antigen-induced peritonitis was as described previously (8). Mice were immunized (day -21) with mBSA (Sigma-Aldrich), emulsified in CFA, intradermally (i.d.) in the base of the tail. A booster injection was given at day -7 . On day 0 , mice were challenged i.p. with $200 \mu \mathrm{g}$ mBSA, and 4 days later, peritoneal exu- 
A

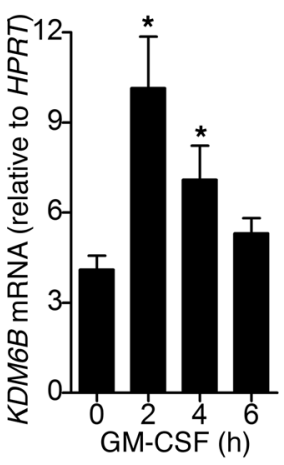

D

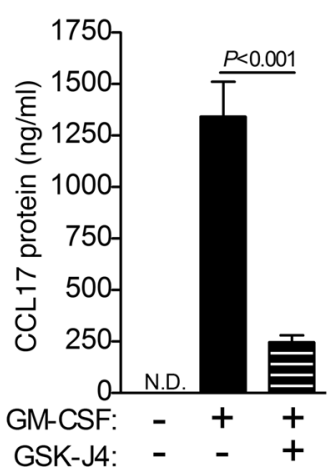

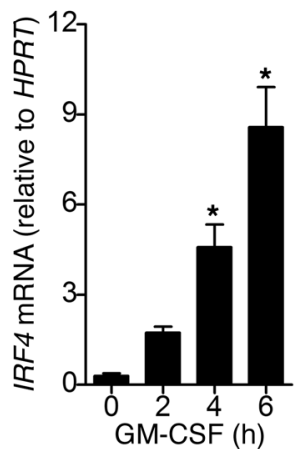

E

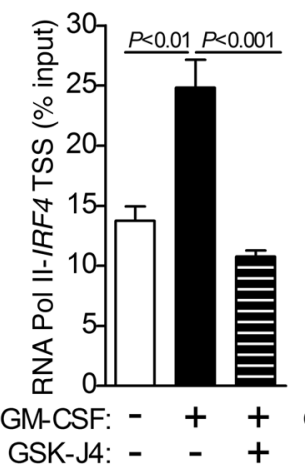

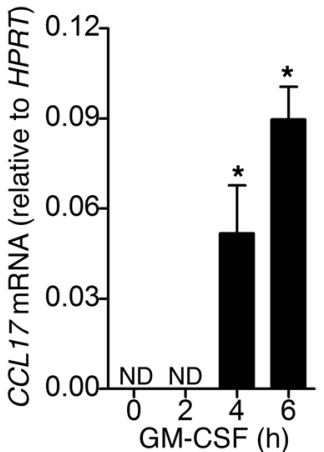

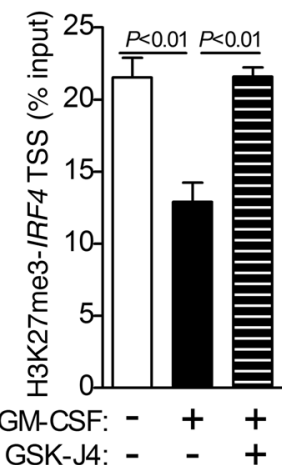

B
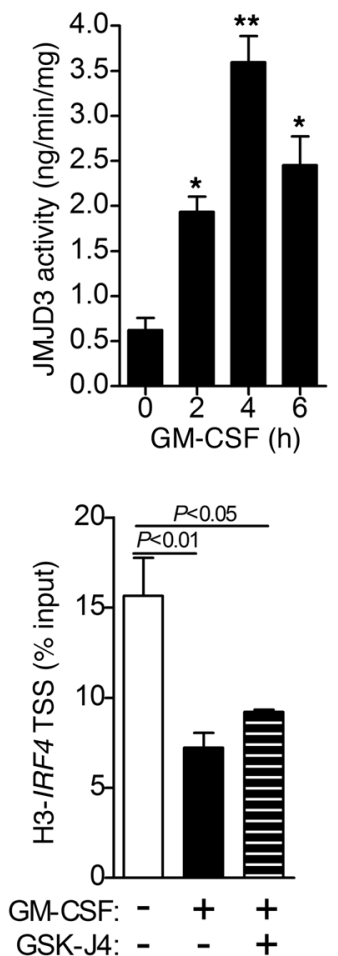

C

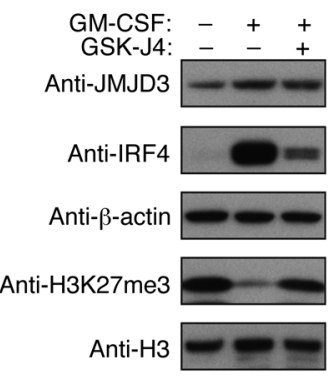

$\mathbf{F}$

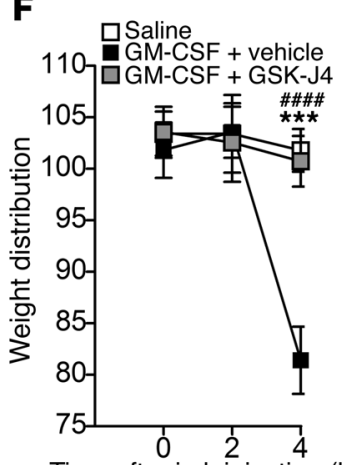

Time after i.pl. injection (h)

Figure 10. GM-CSF regulates IRF4 expression via JMJD3 demethylase in human monocytes, and inhibition of JMJD3 demethylase ameliorates GM-CSFdriven inflammatory pain. (A and B) Human monocytes were treated with GM-CSF (10 ng/ml) for indicated periods of time. (A) KDM6B, IRF4, and CCL17 mRNA expression ( $(P C R)$ and (B) JMJD3 activity were determined $(n=5)$. (C and D) Human monocytes were pretreated with JMJD3 inhibitor GSK-J4 (10 nM) or DMSO for 30 minutes before treatment with GM-CSF $(10 \mathrm{ng} / \mathrm{ml})$ for 16 hours. (C) Whole cell lysates were subjected to Western blotting with anti-JMJD3, anti-IRF4, anti- $\beta$-actin, anti-H3K27me3, and anti-H3 antibodies and (D) secreted CCL17 measured by ELISA $(n=4)$. (E) Human monocytes were pretreated with JMJD3 inhibitor GSK-J4 (10 nM) or DMSO for 30 minutes before treatment with GM-CSF $(10 \mathrm{ng} / \mathrm{ml})$ for 1 hour. ChIP analysis of the association of RNA Pol II, H3K27me3, and total H3 with the IRF4 TSS is expressed as percentage of input DNA $(n=4)$. (F) i.pl. injection of GM-CSF (20 ng) or saline in WT mice treated with/without GSK-J4 (25 mg/kg i.p. at $t=-30$ minutes). Pain development was measured ( $n=11$ per group). Graphs are plotted as mean \pm SEM. $P$ values were obtained using a 1-way ANOVA test (A, B, D, and E) and a 2 -way ANOVA test (F). ${ }^{*} P<0.05 ;{ }^{* *} P<0.01$, vs. $t=0$; ${ }^{* *} P<0.001$, saline vs.

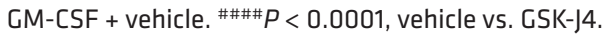

date cells were harvested. For antibody blockade experiments, mice received $150 \mu \mathrm{g}$ anti-GM-CSF $\mathrm{mAb}$ (22E9) or isotype control $\mathrm{mAb}$ (GL117.41) (a gift from J. Abrams, Schering BioPharma) on days 1 and 2 after i.p. challenge (8).

Inflammatory pain models. Inflammatory pain was induced by a single i.pl. injection (10 $\mu \mathrm{l}$ ) of either GM-CSF (20 ng, R\&D Systems), CCL17 (50 ng, R\&D Systems), CFA (Difco), or zymosan (100 $\mu$ g, SigmaAldrich) into the left hind footpad. Saline was used as a control. Paw swelling was measured using spring callipers (Mitutoyo). Indomethacin $(12.5 \mu \mathrm{g} / \mathrm{paw}$, i.pl., Sigma-Aldrich) was given at either 0 or 2 hours after the inciting stimulus injection. The COX2 inhibitor SC58125 (5 $\mathrm{mg} / \mathrm{kg}$, i.p., Tocris) and the JMJD3 inhibitor GSK-J4 (25 mg/kg, i.p., Santa Cruz Biotechnology Inc.) were given at -30 minutes prior to the inciting stimulus injection. Anti-CCL17 mAb (MAB529, clone 110904, R\&D Systems) or isotype control mAb (GL117.41, Schering BioPharma) was given i.pl. $(2 \mu \mathrm{g})$ at the same time as the indicated stimulus.

Pain readings. As an indicator of pain, the differential distribution of weight over a 3-second period between the inflamed paw or limb relative to the noninflamed paw or limb was measured using an incapacitance meter (IITC Life Science Inc.) as before $(1,3,63)$. This technique has previously been validated for measurement of both paw and arthritic knee pain $(1,3,63)$. Mice were acclimatized to the incapacitance meter on at least 3 occasions prior to the commencement of the experiment. Three measurements were taken for each time point and averaged.

mBSA-induced arthritis models. Monoarticular arthritis was induced as before (3) by i.a. injection of $100 \mu \mathrm{g}$ mBSA in $10 \mu \mathrm{l}$ saline into the right knee on day 0 , the left knee being injected with saline, followed by an s.c. injection in the scruff of the neck on days 0 to 2 of either GM-CSF (500 ng, R\&D Systems), CCL17 (600 ng, R\&D Systems), or saline. Mice were sacrificed (day 7) and knee joints collected for histology. Indomethacin ( $1 \mathrm{mg} / \mathrm{kg}$, i.p.) was given once pain was evident.

Zymosan-induced arthritis model. For the induction of the zymosan-induced arthritis model (64), mice were injected with $300 \mu \mathrm{g}$ of sonicated zymosan (Sigma-Aldrich) in a $10 \mu$ l volume into the left knee joint, while the contralateral knee received saline as a control. On day 7, arthritic joints were collected for gene expression analysis using quantitative PCR (qPCR) and histological analysis. Indomethacin (1 mg/kg, i.p., Sigma) was given once pain was evident.

AIA. As before, mice were immunized (day 0) with Sigma-Aldrich, emulsified in CFA, i.d. in the base of the tail (3). Arthritis was induced 7 days later by an i.a. injection of mBSA into the right knee, the left knee being injected with saline. 
Histology. At termination, the knee joints were removed, fixed, decalcified, and paraffin embedded $(1,3)$. Frontal sections $(5 \mu \mathrm{m})$ were stained with H\&E. For the mBSA/GM-CSF and mBSA/CCL17 models, cellular infiltration, synovitis (synovial hyperplasia), pannus formation, cartilage damage, and bone erosions were scored separately from 0 (normal) to 5 (severe) as described previously (3). For zymosan-induced arthritis, cell infiltration, synovial hyperplasia, proteoglycan loss (Safranin O/fast green stain), and bone erosions were scored separately from 0 (normal) to 3 (severe) (64). For AIA, cell infiltration, proteoglycan loss (Safranin O/fast green stain), and bone erosions were scored separately from 0 (normal) to 3 (severe) (3).

Western blotting. Western blotting was performed as described previously (31). Briefly, whole cell extracts were lysed with RIPA buffer. Protein concentrations were determined with a Bio-Rad protein assay kit. Equal amounts of whole cell lysates were run on 10\% NuPAGE (Life Technologies). The separated proteins were transferred onto a PVDF membrane and then Western blotted with appropriate Abs. Abs were against IRF4 (clone M-17) and IRF5 (clone 10T1) (Santa Cruz Biotechnology Inc.); JMJD3 (clone 07-1534), histone H3 (clone A3S), and H3K27me3 (clone 07-449) (Merck Millipore); Hsp90 (clone 68/ Hsp90, BD Biosciences); and $\beta$-actin (clone AC-74, Sigma-Aldrich).

Gene silencing. Human monocytes were nucleofected with IRF4, IRF5, or a nontargeting control siRNA (GE Dharmacon) with Amaxa Kit (Lonza) according to the manufacturer's instructions. The cells were then cultured for another 16 hours in the presence of GM-CSF before analysis.

Flow cytometry. Basal CCL17 and IRF4 expression in GM-BMM and BMM were determined by utilizing $\mathrm{Ccl1} 7^{\mathrm{E} / \mathrm{+}}$ mice and intracellular/ nuclear staining of IRF4 (clone M-17) using the BD Cytofix/Cytoperm Kit, as per the manufacturer's instructions (BD Biosciences). Analysis of inflammatory peritoneal exudate cell populations in $\mathrm{Ccl1}^{\mathrm{E} /+}$ mice was as previously described (8). For analysis of inflammatory peritoneal exudate cell populations in $\mathrm{Ccl1}^{\mathrm{E} / \mathrm{+}}$ mice, cells were stained with Fc block anti-CD16/32 (clone 2.4G2) and fluorochrome-conjugated mAbs specific for mouse MHCII (clone M5/114.15.2)(BioLegend), CD11b (clone M1/70), CD11c (clone HL3), CD115 (clone AFS98), Ly6G (clone 1A8), and the corresponding isotype controls, (BD Biosciences) (8). The cells were analyzed using a CyAn ADP Flow Cytometer (Beckman Coulter). All analyses were performed using Kaluza v1.2 (Beckman Coulter).

$J M J D 3$ activity assay. Human monocytes were lysed following treatment with GM-CSF, and nuclear fractions were enriched with NE-PER Nuclear and cytoplasmic extraction reagents (Thermo Fisher Scientific Inc.). $10 \mu \mathrm{g}$ of nuclear extracts was subjected to the JMJD3 activity assay with a 120-minute incubation period, using a colorimetric Epigenase JMJD3 Demethylase Activity Assay Kit (Epigentek). The demethylated product was ascertained from OD at $450 \mathrm{~nm}$ using a standard curve, and JMJD3 activity (ng/min/mg) was calculated as demethylated product (ng) divided by incubation time ( $\mathrm{min}$ ) and input nuclear extract (mg).

ChIP assay. Human monocytes were treated with GM-CSF (10 ng/ $\mathrm{ml}$ ) for 1 hour before crosslinking protein-DNA complexes with $1 \%$ formaldehyde for 10 minutes at room temperature. ChIP was performed using a ChIP Assay Kit (17-295, Millipore) as per the manufacturer's instructions with the following modifications. DNA was sheared with
Bioruptor XL (Diagenode) with a HI pulse setting and 30 seconds on and 30 seconds off pulses. The cycle was repeated 30 times, resulting in a sonication time of 30 minutes in total to achieve chromatin fragments of 200 to 1,200 base pairs. The lysates were incubated with $1 \mu \mathrm{g}$ of antiRNA Pol II (0.5-952, Millipore), anti-H3K27me3 (07-449, Millipore), or anti-histone H3 (05-925, Millipore) antibodies. Following immunoprecipitation and reversal of DNA-protein crosslinking, as per the manufacturer's instructions, DNA was purified with the MinElute PCR purification kit (QIAGEN). The real-time PCR reaction was then performed on the immunoprecipitated DNA fragments with a SensiFAST SYBR Hi-ROX Kit (Bioline) and the following human IRF4 TSS-specific primers: forward 5'-CCACCTCGCACTCTCAGTTT-3' and reverse 5'-CTGGAGGTCGAACCTCTGGT-3'. Enrichment of histones and RNA Pol II at IRF4 TSS was expressed as percentage of input DNA.

Statistics. For mRNA and protein expression, a 2-tailed Student's $t$ test, a Mann-Whitney $U$ test, or a 2-way ANOVA was used. For pain readings, a 2-tailed Student's $t$ test or 2-way ANOVA was used, and for histologic scores, the Mann-Whitney $U$ 2-sample rank test, 1-way ANOVA, or 2-way ANOVA was used (GraphPad software, version 5.04). Bonferroni's post-hoc test was used when appropriate. Data were plotted as mean \pm SEM with significance as indicated. A $P$ value of less than 0.05 was considered significant.

Study approval. All animal experiments were approved by The University of Melbourne Animal Ethics committee.

\section{Author contributions}

Conceptualization was by AA, AD Cook, and JAH. Investigation was by AA, AD Cook, MCL, RS, HWK, MWNC, CL, AJF, DCL, AD Christensen, ATF, PYL, HK, KN, and NS. Resources were provided by NS, IF, SLN, and SJT. Formal analysis was by AA, AD Cook, and MO. AA, AD Cook, and JAH wrote the original draft. Writing-review and editing were by AA, AD Cook, JAH, IF, SLN, and SJT. Supervision was by AA, AD Cook, and JAH.

\section{Acknowledgments}

AA and JAH were supported in part by a grant from GSK. AD Cook and JAH were supported by a grant (1043147) and JAH by a Senior Principal Research Fellowship from the National Health and Medical Research Council of Australia. AD Christensen was supported by a postdoc fellowship from Novo Nordisk A/S, Denmark. NS and IF were supported by the Deutsche Forschungsgemeinschaft (DFG) through SFB704. IF is a member of the DFG-funded cluster of excellence ImmunoSensation. SLN was supported by grants (361646, 575500 and 1054925) from the National Health and Medical Research Council of Australia. SJT was supported by grants (5671222 and 1003131) from the National Health and Medical Research Council of Australia and by an Australian Research Council Future Fellowship.

Address correspondence to: John A. Hamilton, Department of Medicine, Royal Melbourne Hospital, University of Melbourne, Parkville, Victoria 3050, Australia. Phone: 61.3.8344.5480; E-mail: jahami@unimelb.edu.au.
1. Cook AD, et al. Granulocyte-macrophage colonystimulating factor is a key mediator in experimental osteoarthritis pain and disease development. Arthritis Res Ther. 2012;14(5):R199.
2. Schweizerhof M, et al. Hematopoietic colony-stimulating factors mediate tumor-nerve interactions and bone cancer pain. Nat Med. 2009;15(7):802-807.
3. Cook AD, et al. Granulocyte-macrophage colony-stimulating factor is a key mediator in inflammatory and arthritic pain. Ann Rheum Dis. 2013;72(2):265-270. 
4. Hamilton JA. GM-CSF as a target in inflammatory/autoimmune disease: current evidence and future therapeutic potential. Expert Rev Clin Immunol. 2015;11(4):457-465.

5. Hamilton JA. Colony-stimulating factors in inflammation and autoimmunity. Nat Rev Immunol. 2008;8(7):533-544.

6. Hamilton JA, Achuthan A. Colony stimulating factors and myeloid cell biology in health and disease. Trends Immunol. 2013;34(2):81-89.

7. Ko HJ, et al. GM-CSF-responsive monocytederived dendritic cells are pivotal in Th17 pathogenesis. J Immunol. 2014;192(5):2202-2209.

8. Louis $\mathrm{C}$, et al. Specific contributions of CSF-1 and GM-CSF to the dynamics of the mononuclear phagocyte system. JImmunol. 2015;195(1):134-144.

9. Alferink J, et al. Compartmentalized production of CCL17 in vivo: strong inducibility in peripheral dendritic cells contrasts selective absence from the spleen. J Exp Med. 2003;197(5):585-599.

10. Iellem A, et al. Unique chemotactic response profile and specific expression of chemokine receptors CCR 4 and CCR 8 by CD4(+)CD25(+) regulatory T cells. JExp Med. 2001;194(6):847-853.

11. Katakura T, Miyazaki M, Kobayashi M, Herndon DN, Suzuki F. CCL17 and IL-10 as effectors that enable alternatively activated macrophages to inhibit the generation of classically activated macrophages. JImmunol. 2004;172(3):1407-1413.

12. Stutte S, et al. Requirement of CCL17 for CCR7- and CXCR4-dependent migration of cutaneous dendritic cells. Proc Natl Acad Sci USA. 2010;107(19):8736-8741.

13. Heiseke AF, et al. CCL17 promotes intestinal inflammation in mice and counteracts regulatory T cell-mediated protection from colitis. Gastroenterology. 2012;142(2):335-345.

14. Yoshie O, Matsushima K. CCR4 and its ligands: from bench to bedside. Int Immunol. 2015;27(1):11-20.

15. Oh SB, Tran PB, Gillard SE, Hurley RW, Hammond DL, Miller RJ. Chemokines and glycoprotein120 produce pain hypersensitivity by directly exciting primary nociceptive neurons. J Neurosci. 2001;21(14):5027-5035.

16. Weber C, et al. CCL17-expressing dendritic cells drive atherosclerosis by restraining regulatory $\mathrm{T}$ cell homeostasis in mice. JClin Invest. 2011;121(7):2898-2910.

17. Okamoto H, Koizumi K, Yamanaka H, Saito T, Kamatani N. A role for TARC/CCL17, a CC chemokine, in systemic lupus erythematosus. JRheumatol. 2003;30(11):2369-2373.

18. Kataoka Y. Thymus and activation-regulated chemokine as a clinical biomarker in atopic dermatitis. J Dermatol. 2014;41(3):221-229.

19. Staples KJ, Hinks TS, Ward JA, Gunn V, Smith C, Djukanović R. Phenotypic characterization of lung macrophages in asthmatic patients: overexpression of CCL17. J Allergy Clin Immunol. 2012;130(6):1404-12.e7.

20. Belperio JA, et al. The role of the Th2 CC chemokine ligand CCL17 in pulmonary fibrosis. JImmunol. 2004;173(7):4692-4698.

21. Tamura T, Yanai H, Savitsky D, Taniguchi T. The IRF family transcription factors in immunity and oncogenesis. Annu Rev Immunol. 2008;26:535-584.
22. Lehtonen A, et al. Differential expression of IFN regulatory factor 4 gene in human monocytederived dendritic cells and macrophages. J Immunol. 2005;175(10):6570-6579.

23. Schlitzer A, et al. IRF4 transcription factordependent $C D 11 b+$ dendritic cells in human and mouse control mucosal IL-17 cytokine responses. Immunity. 2013;38(5):970-983.

24. Persson EK, et al. IRF4 transcription-factordependent CD103(+)CD11b(+) dendritic cells drive mucosal $\mathrm{T}$ helper 17 cell differentiation. Immunity. 2013;38(5):958-969.

25. Gao Y, et al. Control of T helper 2 responses by transcription factor IRF4-dependent dendritic cells. Immunity. 2013;39(4):722-732.

26. Negishi $\mathrm{H}$, et al. Negative regulation of Toll-like-receptor signaling by IRF-4. Proc Natl Acad Sci USA. 2005;102(44):15989-15994.

27. Honma K, et al. Interferon regulatory factor 4 negatively regulates the production of proinflammatory cytokines by macrophages in response to LPS. Proc Natl Acad Sci USA. 2005;102(44):16001-16006.

28. Eguchi J, Kong X, Tenta M, Wang X, Kang S, Rosen $\mathrm{ED}$. Interferon regulatory factor $4 \mathrm{reg}$ ulates obesity-induced inflammation through regulation of adipose tissue macrophage polarization. Diabetes. 2013;62(10):3394-3403.

29. Akbari M, et al. IRF4 in dendritic cells inhibits IL-12 production and controls Th1 immune responses against Leishmania major. J Immunol. 2014;192(5):2271-2279.

30. Krausgruber T, et al. IRF5 promotes inflammatory macrophage polarization and TH1-TH17 responses. Nat Immunol. 2011;12(3):231-238.

31. Lacey DC, et al. Defining GM-CSF- and macrophage-CSF-dependent macrophage responses by in vitro models. J Immunol. 2012;188(11):5752-5765.

32. Hamilton JA, Whitty GA, Stanton H, Meager A. Effects of macrophage-colony stimulating factor on human monocytes: induction of expression of urokinase-type plasminogen activator, but not of secreted prostaglandin E2, interleukin-6, interleukin-1, or tumor necrosis factor-alpha. J Leukoc Biol. 1993;53(6):707-714.

33. Fleetwood AJ, Lawrence T, Hamilton JA, Cook AD. Granulocyte-macrophage colony-stimulating factor (CSF) and macrophage CSF-dependent macrophage phenotypes display differences in cytokine profiles and transcription factor activities: implications for CSF blockade in inflammation. J Immunol. 2007;178(8):5245-5252.

34. Fleetwood AJ, Dinh H, Cook AD, Hertzog PJ, Hamilton JA. GM-CSF- and M-CSF-dependent macrophage phenotypes display differential dependence on type I interferon signaling. J Leukoc Biol. 2009;86(2):411-421.

35. Yang YH, Hamilton JA. Dependence of interleukin-1-induced arthritis on granulocyte-macrophage colony-stimulating factor. Arthritis Rheum. 2001;44(1):111-119.

36. Meller ST, Gebhart GF. Intraplantar zymosan as a reliable, quantifiable model of thermal and mechanical hyperalgesia in the rat. Eur J Pain. 1997;1(1):43-52.

37. Blom AB, van Lent PL, Holthuysen AE, van den Berg WB. Immune complexes, but not strep- tococcal cell walls or zymosan, cause chronic arthritis in mouse strains susceptible for collagen type II auto-immune arthritis. Cytokine. 1999;11(12):1046-1056.

38. Satoh T, et al. The Jmjd3-Irf4 axis regulates M2 macrophage polarization and host responses against helminth infection. Nat Immunol. 2010;11(10):936-944.

39. Verreck FA, et al. Human IL-23-producing type 1 macrophages promote but IL-10-producing type 2 macrophages subvert immunity to (myco)bacteria. Proc Natl Acad Sci U S A. 2004;101(13):4560-4565.

40. Croxford AL, et al. The cytokine GM-CSF drives the inflammatory signature of CCR2+ monocytes and licenses autoimmunity. Immunity. 2015;43(3):502-514.

41. Globisch T, et al. Cytokine-dependent regulation of dendritic cell differentiation in the splenic microenvironment. Eur JImmunol. 2014;44(2):500-510.

42. Liddiard K, Welch JS, Lozach J, Heinz S, Glass CK, Greaves DR. Interleukin-4 induction of the CC chemokine TARC (CCL17) in murine macrophages is mediated by multiple STAT 6 sites in the TARC gene promoter. BMC Mol Biol. 2006;7:45.

43. Crapster-Pregont M, Yeo J, Sanchez RL, Kuperman DA. Dendritic cells and alveolar macrophages mediate IL-13-induced airway inflammation and chemokine production. J Allergy Clin Immunol. 2012;129(6):1621-7.e3.

44. Kitajima M, Ziegler SF. Cutting edge: identification of the thymic stromal lymphopoietinresponsive dendritic cell subset critical for initiation of type 2 contact hypersensitivity. JImmunol. 2013;191(10):4903-4907.

45. Martinez FO, Helming L, Gordon S. Alternative activation of macrophages: an immunologic functional perspective. Annu Rev Immunol. 2009;27:451-483.

46. Murray PJ, et al. Macrophage activation and polarization: nomenclature and experimental guidelines. Immunity. 2014;41(1):14-20.

47. Lari R, et al. Macrophage lineage phenotypes and osteoclastogenesis--complexity in the control by GM-CSF and TGF-beta. Bone. 2007;40(2):323-336.

48. Helft J, et al. GM-CSF Mouse Bone Marrow Cultures Comprise a Heterogeneous Population of CD11c(+)MHCII(+) Macrophages and Dendritic Cells. Immunity. 2015;42(6):1197-1211.

49. Gordon S, Mantovani A. Diversity and plasticity of mononuclear phagocytes. Eur J Immunol. 2011;41(9):2470-2472.

50. Willart MA, et al. Interleukin-1 $\alpha$ controls allergic sensitization to inhaled house dust mite via the epithelial release of GM-CSF and IL-33. J Exp Med. 2012;209(8):1505-1517.

51. De Santa F, et al. Jmjd 3 contributes to the control of gene expression in LPS-activated macrophages. EMBO J. 2009;28(21):3341-3352.

52. Gomez-Rodriguez J, et al. Itk is required for Th9 differentiation via TCR-mediated induction of IL-2 and IRF4. Nat Commun. 2016;7:10857.

53. Walker K, et al. Metabotropic glutamate receptor subtype 5 (mGlu5) and nociceptive function. I. Selective blockade of mGlu5 receptors in models 
of acute, persistent and chronic pain. Neuropharmacology. 2001;40(1):1-9.

54. Xu WD, Firestein GS, Taetle R, Kaushansky K, Zvaifler NJ. Cytokines in chronic inflammatory arthritis. II. Granulocyte-macrophage colony-stimulating factor in rheumatoid synovial effusions. JClin Invest. 1989;83(3):876-882.

55. Hampel U, Sesselmann S, Iserovich P, Sel S, Paulsen F, Sack R. Chemokine and cytokine levels in osteoarthritis and rheumatoid arthritis synovial fluid. JImmunol Methods. 2013;396(1-2):134-139.

56. Moret FM, et al. Intra-articular CD1c-expressing myeloid dendritic cells from rheumatoid arthritis patients express a unique set of $\mathrm{T}$ cell-attracting chemokines and spontaneously induce Th1, Th17 and Th2 cell activity. Arthritis Res Ther.
2013;15(5):R155.

57. Mittrücker HW, et al. Requirement for the transcription factor LSIRF/IRF4 for mature $\mathrm{B}$ and $\mathrm{T}$ lymphocyte function. Science. 1997;275(5299):540-543.

58. Mombaerts P, Iacomini J, Johnson RS, Herrup K, Tonegawa S, Papaioannou VE. RAG-1-deficient mice have no mature B and T lymphocytes. Cell. 1992;68(5):869-877.

59. Gagnon-Bartsch JA, Speed TP. Using control genes to correct for unwanted variation in microarray data. Biostatistics. 2012;13(3):539-552.

60. Jacob L, Gagnon-Bartsch JA, Speed TP. Correcting gene expression data when neither the unwanted variation nor the factor of interest are observed. Biostatistics. 2016;17(1):16-28.
61. Eisenberg E, Levanon EY. Human housekeeping genes, revisited. Trends Genet. 2013;29(10):569-574.

62. Brazma A, et al. Minimum information about a microarray experiment (MIAME)-toward standards for microarray data. Nat Genet. 2001;29(4):365-371.

63. Inglis JJ, et al. Regulation of pain sensitivity in experimental osteoarthritis by the endogenous peripheral opioid system. Arthritis Rheum. 2008;58(10):3110-3119.

64. Keystone EC, Schorlemmer HU, Pope C, Allison AC. Zymosan-induced arthritis: a model of chronic proliferative arthritis following activation of the alternative pathway of complement. Arthritis Rheum. 1977;20(7):1396-1401. 